First publ. in: Graphene nanoelectronics : metrology, synthesis, properties and applications /

\title{
Epitaxial Graphene on Metals
}

\author{
Yuriy Dedkov, Karsten Horn, Alexei Preobrajenski, and Mikhail Fonin
}

\begin{abstract}
In this chapter, we review the recent results on graphene films epitaxially grown on $3 d-5 d$ transition-metal surfaces focussing mainly on their atomic structure, aspects of chemical interaction both with the substrate and with adsorbates and the electronic structure of graphene at the interface. We discuss a possibility to prepare sandwich-like structures in the form of intercalates as a possible way to controllably tailor the electronic properties of graphene. Recent works on the templated growth of metallic nanostructures on graphene moiré are also reviewed.
\end{abstract}

\subsection{Introduction}

Graphene is a flat single layer of carbon atoms arranged in a honeycomb lattice with two crystallographically equivalent atoms (A and B) in its primitive unit cell [1-3] [Fig. 7.1a]. The $s p^{2}$ hybridization between one $2 s$ orbital and two $2 p$ orbitals leads to a trigonal planar structure with a formation of strong $\sigma$ bonds between carbon atoms that are separated by $1.42 \AA$. The bonding $\sigma$ orbitals have a filled shell and, hence, form deeper valence band levels. The $2 p_{z}$ orbitals on the neighboring carbon atoms are perpendicular to the planar structure of the graphene layer and can bind covalently, leading to the formation of a $\pi$ band. The unique property

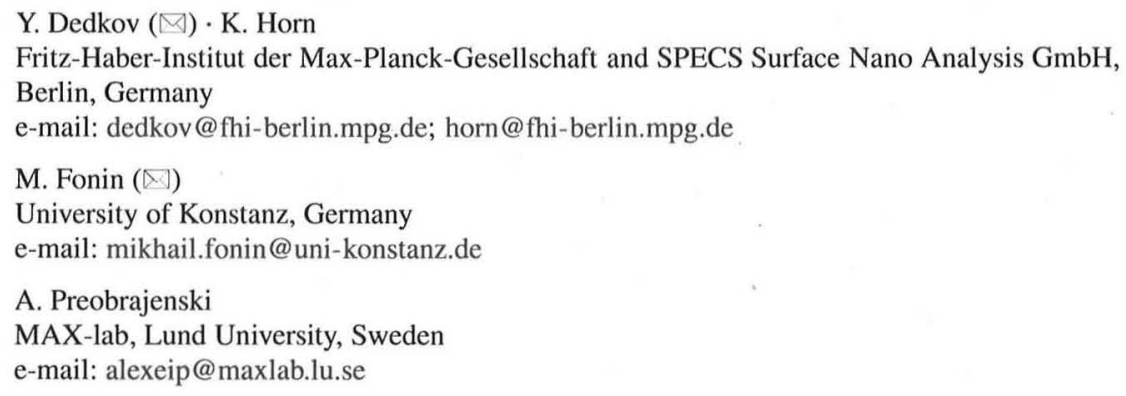



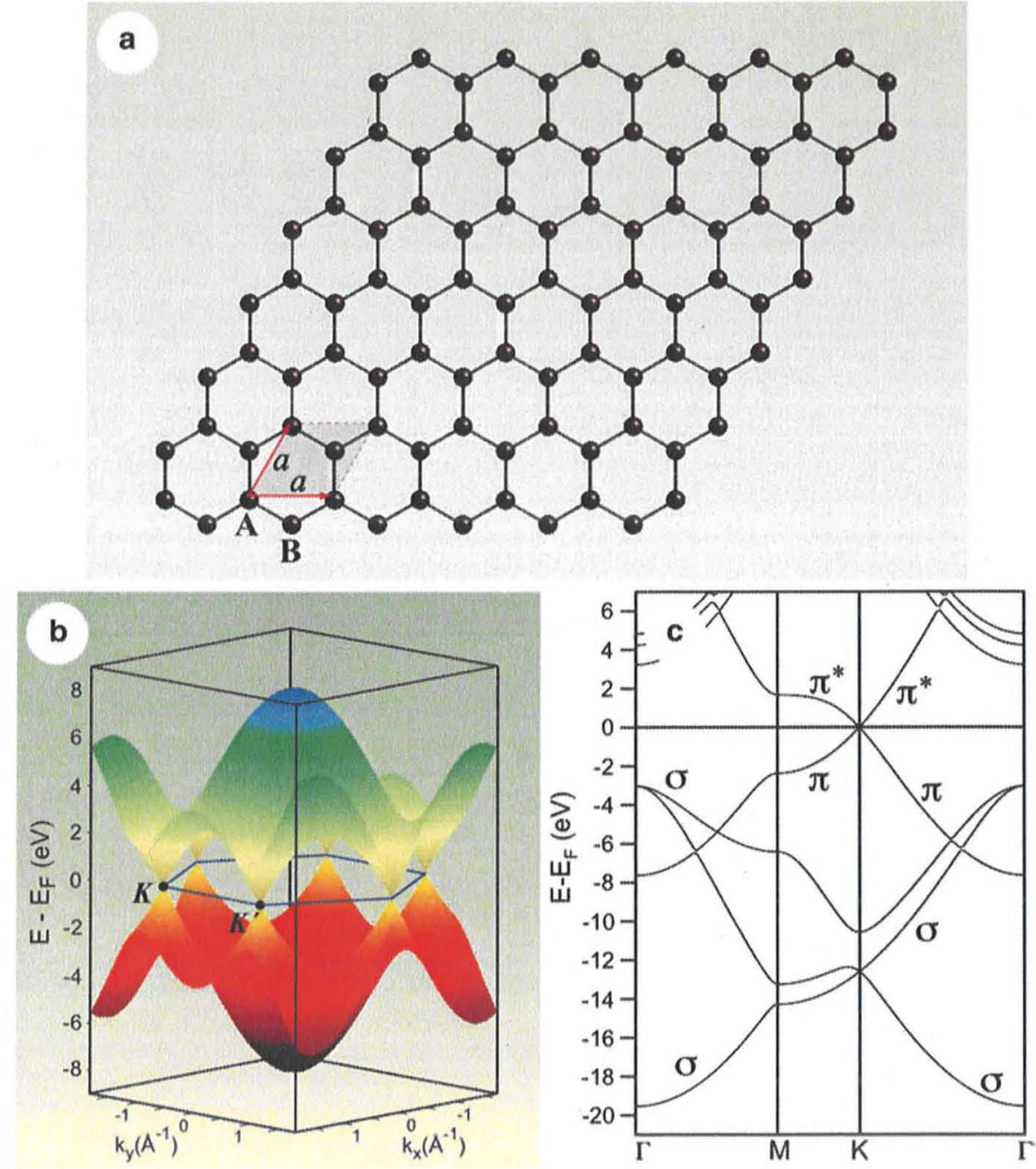

Fig. 7.1 (a) Crystal structure of the graphene layer, where carbon atoms are arranged in a honeycomb lattice. The unit cell of graphene with lattice constant $a$ has two carbon atoms per unit cell, A and B. (b) Electronic dispersion of $\pi$ and $\pi^{*}$ states in the honeycomb lattice of free-standing graphene obtained in the framework of tight-binding approach. These branches have linear dispersion in the vicinity of the $K$ points of the Brillouin zone of graphene. (c) Band structure of free-standing graphene as obtained by means of DFT $\left(\sigma, \pi\right.$, and $\pi^{*}$ bands are marked)

of the electronic structure of graphene is that the $\pi$ and $\pi^{*}$ bands touch at a single point at the Fermi energy $\left(E_{\mathrm{F}}\right)$ at the corner of graphene's hexagonal Brillouin zone, and close to this so-called Dirac point the bands display a linear dispersion and form Dirac cones [2] [Fig. 7.1b]. Thus, undoped graphene is a semimetal ("zerogap semiconductor"). The linear dispersion of the bands mimics the physics of 
quasiparticles with zero mass, the so-called massless Dirac fermions [1-3]. The fascinating electronic and transport properties of graphene [1-3] make it to a point of focus not only in fundamental research but also in applied science and technology with a vision to implement graphene in a myriad of electronic devices replacing the existing silicon technology.

However, a widespread implementation of graphene in electronics has been hampered by the two major difficulties: reliable production of high-quality samples, especially in a large-scale fashion and the "zero-gap" electronic structure of graphene, which leads to limitations for direct application of this material in possible electronic devices. As a response to the first challenge, a number of approaches for single layer graphene preparation have been tested, corroborating chemical vapor deposition (CVD) on transition-metal surfaces to be the most promising alternative to micromechanical cleavage for producing macroscopic graphene films. In 2008, mass-production of continuous graphene wafers by the CVD method on polycrystalline $\mathrm{Ni}$ or $\mathrm{Cu}$ surfaces and its transfer to arbitrary substrates was demonstrated [4-6]. The transferred graphene films show very low sheet resistance of $280 \Omega$ per square, with $80 \%$ optical transparency, high electron mobility of $3,700 \mathrm{~cm}^{2} \mathrm{~V}^{-1} \mathrm{~s}^{-1}$, and the half-integer quantum Hall effect at low temperatures, indicating that the quality of graphene grown by CVD is as high as that of mechanically cleaved graphene. Further modifications of this method within less than one year led to even more fascinating results, where graphene layers as large as 30 inches were transferred on to a polymer film for the preparation of transparent electrodes [7]. Other recent examples of CVD graphene growth on polycrystalline metal substrates include, for example, $\mathrm{Pt}$ [8] and $\mathrm{Ru}$ [9] substrates.

Despite the fascinating recent achievements in graphene production, the second problem of controllable doping should be solved prior to being able to implement graphene in any kind of electronic device. Several strategies exist, which allow the modification of the electronic structure of graphene, including (1) fabrication of narrow, straight-edged stripes of graphene, so-called nanoribbons [10,11], (2) preparation of bi-layer graphene $[12,13],(3)$ direct chemical doping of graphene by an exchange of a small amount of carbon atoms by nitrogen [14], boron [15] or transition-metal atoms [16]; (4) modification of the electronic structure of the graphene by interaction with substrates [17-21]; (5) intercalation of materials underneath graphene prepared on different substrates [22-27]; (6) deposition of different materials on top of graphene [28-30]. Not surprisingly, the investigation of graphene interaction with supporting or doping materials, which may provide an additional important degree of control of graphene properties has become one of the most important research fields [31-34].

Focusing on the graphene/ferromagnet systems, such hybrid structures may have further intriguing applications. Recent theoretical calculations by Karpan and co-workers $[35,36]$ for graphene/metal interfaces imply the possibility of an ideal spin filtering in the current-perpendicular-to-the-plane (CPP) configuration for ferromagnet/graphene/ferromagnet sandwich-like structures [Fig. 7.2a]. In this work, the close-packed surfaces of $\mathrm{Co}$ and $\mathrm{Ni}$ were considered as ferromagnetic (FM) electrodes, which perfectly coincide with graphene from the crystallographic 


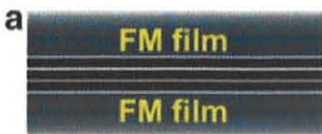

c.

\section{Substrate}

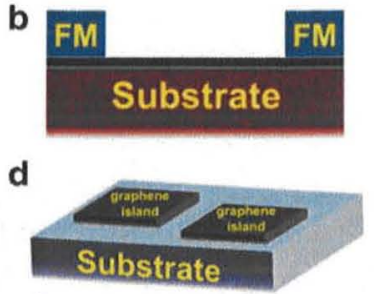

Fig. 7.2 Schematic representations of graphene-based systems, which are of interest for the present graphene/metal studies: (a) spin-filtering device, (b) graphene-based spin-FET, (c) clusters on graphene "nanomesh," and (d) graphene islands ("nanodots") on metallic substrates

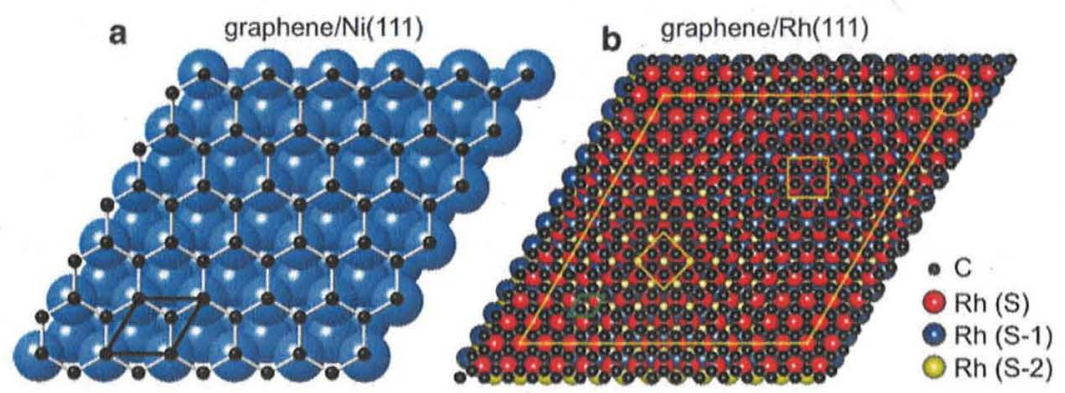

Fig. 7.3 In (a), top-view of a simple ball model for the top-fcc graphene/Ni(111) system is shown. Carbon atoms are small black spheres and nickel atoms are big blue spheres. In (b), top-view of a simple ball model for the growth of graphene on $\mathrm{Rh}(111)$ is shown. Carbon atoms are black, first-layer Rh atoms are red, second-layer Rh atoms are blue, and third-layer Rh atoms are yellow spheres. The yellow rhombus outlines the supercell of the moiré structure with four different positions: atop (marked by a circle), top-fcc (marked by a square), and top-hcp (marked by a rotated square)

point of view [Fig. 7.3a]. The spin-filtering effect originates form the unique overlapping of the electronic structures of the graphene monolayer and the closepacked surfaces of ferromagnetic $\mathrm{Ni}$ and Co. Graphene is a semimetal with electronic density in the vicinity of $E_{\mathrm{F}}$ at the corners ( $K$ points) of the hexagonal Brillouin zone [Fig. 7.1b]. If the Fermi surface projections of ferromagnetic metals, $f c c \mathrm{Ni}$ or $\mathrm{Co}$, on the (111) close-packed plane are considered, then in both cases graphene has only a minority electron density around the $K$ points of the surface Brillouin zone. In the absence of any additional factors that lower the symmetry of the system, the preferential transport of only minority electrons and thus perfect spin-filtering will occur in an FM/graphene/FM stack $[35,36]$. The interaction between graphene and ferromagnetic materials will, however, change the electronic properties of the interface partially quenching the spin-filtering, but a sizable effect can still be detected by choosing a proper combination of FM materials [37]. The spin-filtering effect is also predicted to increase strongly when multilayer graphene is used in the junctions [35,36] or when $\mathrm{Cu}$ or $\mathrm{Mn}$ decouple graphene from FM [36, 37], making these systems highly attractive for possible applications as 
spintronic units. Moreover, graphene on its own might be a material of choice for the realization of spintronic devices [38], e. g. the spin-field-effect transistor (spin-FET) [Fig. 7.2b]. Such systems usually require the effective injection of spin-polarized electrons in the conductive channel, which can be made from graphene [39]. Hence, a comprehensive knowledge about the electronic and magnetic properties of graphene/FM interfaces is important for the fabrication of effective graphene-based spintronic units.

Beyond the utilization of its unique electronic properties, graphene may also be exploited for many other applications, for example, using graphene layers as a template for the growth of regular arrays of nanostructures [Fig. 7.2c]. On closepacked noble metal surfaces, such as $\operatorname{Ir}(111)$ [40], $\operatorname{Rh}(111)$ [41] or $\operatorname{Ru}(0001)$ [4147], graphene forms periodically corrugated moiré patterns with a period of several nanometers [Fig. 7.3b], and such superstructures can act as templates for the preparation of exceptionally well-ordered nanocluster lattices [40, 48-51]. Monodisperse metal cluster arrays on the inert graphene surface can be used in catalysis or magnetic data storage.

In this chapter, we give a comprehensive overview of the recent studies of graphene on metals focussing on such important aspects as morphology and structure of graphene on metals, and the influence of the geometrical arrangement of carbon atoms on the electronic structure of graphene/metal systems. We review results obtained mostly by surface sensitive techniques including scanning tunneling microscopy (STM), core-hole spectroscopies [core-level photoelectron spectroscopy (PES) as well as near-edge X-ray absorption fine-structure spectroscopy (NEXAFS) and X-ray magnetic circular dichroism (XMCD)], and spin- and angle-resolved photoelectron spectrocopies [(SP)ARPES] studies on the graphene overlayers on latice-matched and lattice-mismatched metallic surfaces. We show and discuss how the graphene-metal interaction depends on the metallic substrate and also discuss the possible ways to modify this interaction. Finally, we briefly discuss future developments of this topic with the possible formation of hybrid structures on the basis of graphene and metal.

\subsection{Methods of Graphene Preparation on Metal Surfaces}

Two common methods of graphene preparation on metallic surfaces exist: (1) elevated temperature segregation of the carbon atoms to the surface of a bulk metallic sample, which was doped with carbon prior to the treatment [Fig. 7.4a] and (2) thermal decomposition of carbon-containing molecules on the surface of transition metals (TMs) [Fig. 7.4b]. In the first method, the transition-metal sample with some amount of carbon impurities or the bulk crystal previously loaded with carbon (via keeping the sample at elevated temperature in the atmosphere of $\mathrm{CO}$ or hydrocarbons) is annealed at higher temperatures. This procedure leads to the segregation of carbon atoms to the surface of the metal. Careful control of the temperature and the cooling rate of the sample allows varying the thicknesses of 


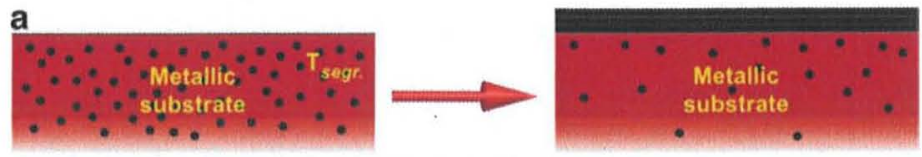

Segregation of carbon from the bulk of metal

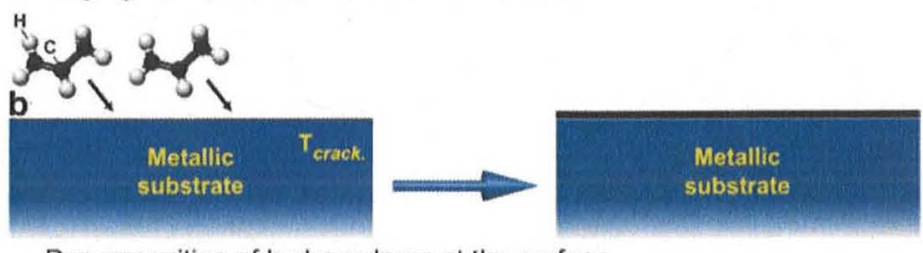

Decomposition of hydrocarbons at the surface

Fig. 7.4 Two ways of the graphene preparation on metal surfaces: (a) Segregation of bulkdissolved carbon atoms to the surface at high temperature $T_{\text {segr }}$; (b) Decomposition (cracking) of hydrocarbon molecules at the surface of transition metals at high temperature $T_{\text {crack }}$

the grown graphene layer: monolayer versus multilayer growth. The second method involves the thermal decomposition (cracking) of carbon-containing molecules at a metal surface. Light hydrocarbon molecules, such as ethylene or propene, are commonly used, but the successful decomposition of $\mathrm{CO}$, acetylene, and of heavy hydrocarbon molecules, such as cyclohexane, $n$-heptane, benzene, and toluene, was also demonstrated [17]. Molecules can be adsorbed on a metal surface at room temperature, and then annealing of the sample leads to the decomposition of molecules and hydrogen desorption. Alternatively, the hot sample surface can be directly exposed to precursor molecules, which decompose at the sample surface. Recent experiments demonstrate that both methods, segregation and decomposition, lead to graphene layers of similar quality. In case of segregation, the kinetics of single graphene layer formation is defined by a careful control of the annealing temperature (but multilayers of graphene could also be prepared). In the second method, the graphene thickness is naturally restricted to a single-layer due to the fact that the chemical reaction on the catalytically active metallic surface takes the place. Thus, the speed of hydrocarbon decomposition drops down by several orders of magnitude as soon as the first graphene monolayer is formed [52, 53]. A comprehensive list of metal surfaces, which were used for the preparation of graphene layers is compiled in [17], where preparation methods, the main experimental methods, and the corresponding references are presented for different lattice-matched and mismatched metallic surfaces.

\subsection{Experimental Methods}

Here, we briefly describe the main microscopic and spectroscopic methods used for the investigation of graphene layers on metal surfaces. For a detailed investigation 

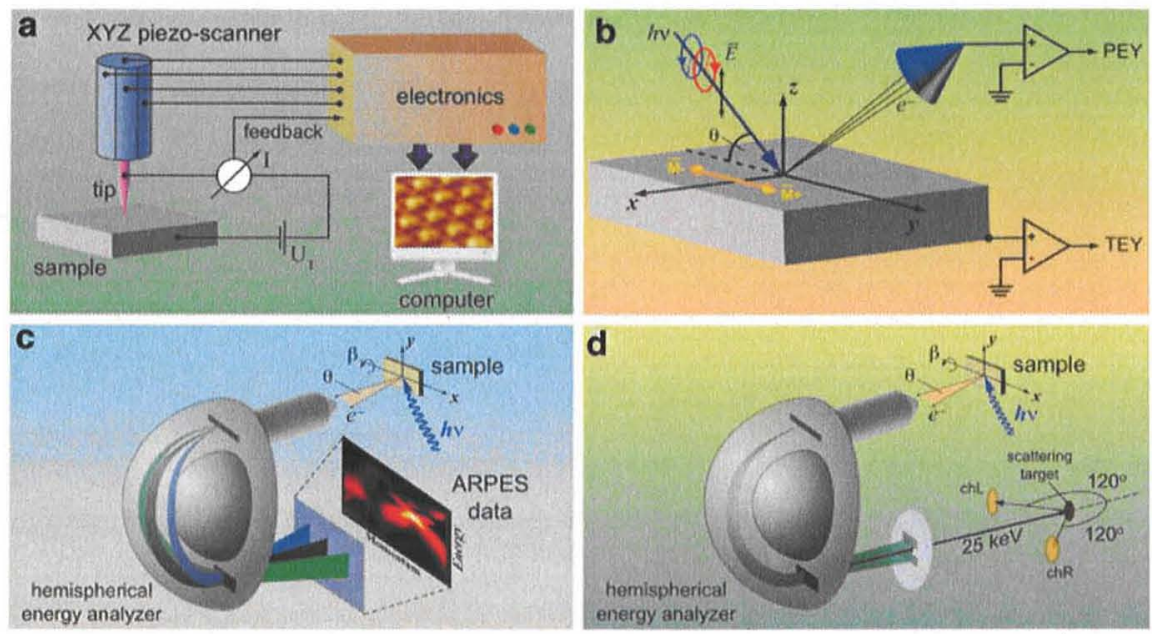

Fig. 7.5 Schematic presentation of the surface science experimental methods mainly referred to in this chapter. (a) In STM via keeping the tunneling current constant while scanning, the surface topography can be imaged with atomic resolution. (b) In NEXAFS or XMCD experiments, the photon energy of incoming linearly or circularly polarized light, respectively, is tuned over a particular absorption edge and total or partial electron yield is measured. (c) In ARPES measurements, the light at a fixed photon energy and polarization is used and photoelectrons are analyzed by their kinetic energy ( $E_{\text {kin }}$ and emission angle $(\theta)$ using a 2D CCD detector allowing to measure the sample electronic structure along a particular direction in the reciprocal space in one shot. Rotation of the sample by an angle $\beta$ produces the 3D data set of experimental photoemission intensity, $I\left(E_{\mathrm{kin}}, k_{x}, k_{y}\right)$, where $k_{x}$ and $k_{y}$ are in-plane components of the wave-vector calculated from the experimental geometry. (d) In spin-resolved PES experiments, 2D CCD detector from (c) is replaced by a spin detector (usually classical or mini Mott type) where spin-separation is performed

of surface topography and atomic arrangement, STM is usually implemented (for details, see [54,55] and Chap.3). This experimental technique based on vacuum tunneling of electrons, nowadays provides unprecedented insight into atomic structures and local electronic structure at surfaces of low-dimensional nano-objects. In an STM experiment, an atomically sharp tip is positioned by piezoelectric actuators above the surface of an electrically conducting sample at a distance of typically $5-15 \AA$ [Fig. 7.5a]. The application of a small voltage $U_{\mathrm{T}}$ between tip and sample results in a quantum tunneling current. By keeping the tunneling current constant while scanning the surface topography can be imaged with atomic resolution. In results presented here, the sign of the bias voltage corresponds to the voltage at the sample. Tunneling parameters are given separately in text for each STM image: $U_{\mathrm{T}}$ for tunneling voltage and $I_{\mathrm{T}}$ for tunneling current.

In order to obtain information about the unoccupied electronic structure above the Fermi level, the NEXAFS spectroscopy is commonly implemented [Fig. 7.5b]. In this method, the energy of photons generated by a synchrotron light source is tuned over a particular absorption threshold, hence promoting core electrons 
to unoccupied states above $E_{\mathrm{F}}$. In this case, the absorption intensity is roughly proportional to the density of the empty valence band states, although distortions due to final-state effects can be considerable. In order to address the average spatial orientation of selected molecular orbitals (for example $\pi$ or $\sigma$ in graphene) at the surface, one can vary the orientation of the sample surface normal with respect to the wave vector of the linearly polarized X-ray light and monitor the absorption intensity. In such an experiment, the absorption intensity associated with a specific molecular orbital final state has a maximum if the electric field vector is aligned parallel to the direction of maximum charge or hole density, i.e. along a molecular orbital, and the intensity vanishes if the electric field vector is perpendicular to the orbital axis. A detailed description of the angular dependence of NEXAFS intensities can be found elsewhere [56, 57]. The XMCD technique is used for the investigation of magnetic properties of materials and allows for a direct determination of spin- and orbital-magnetic moments. In this case, circularly polarized light is used and the absorption coefficient depends on the relative orientation of photon spin and magnetization direction of the sample. The quantitative analysis of the absorption spectra obtained on a magnetic sample with circularly polarized light can be performed with the help of the so-called magnetooptical sum rules for spin- and orbital-magnetic moments [58, 59].

PES represents one of the main experimental tools, which allows to obtain comprehensive information about the electronic structure of solids below $E_{F}$ (occupied valence band states) including the additional information about manyelectron and quasiparticle effects [60-62] (see also Chap.4). In a photoemission experiment, an electron from an initial state $(i)$ below $E_{\mathrm{F}}$ is excited to some final state $(f)$ above the vacuum level with an incident photon $(h v)$. By measuring the kinetic energy of the electron in the final state $E_{f}$ and knowing the incident photon energy $(h \nu)$ and the work function $(\phi)$, the experimenter can trace back to the binding energy of the electron in the initial state $E_{i}$. Electrons which stem from the valence band can be found at binding energies of several $\mathrm{eV}$ while those from core levels contribute between several tens $\mathrm{eV}$ to several thousand $\mathrm{eV}$. Modern electron energy analyzers in ARPES use the so-called 2D CCD detector allowing to obtain the band dispersion of the valence band states in a wide range of emission angles $\theta$ in one shot (at a fixed angle $\beta$ ) [Fig. 7.5c]. The emission angle $\theta$ can be used for the calculation of wave vector component $k_{x}$ of electron in solid. A rotation of the sample by angle $\beta$ produces the $3 \mathrm{D}$ data set of experimental photoemission intensity, $I\left(E_{\text {kin }}, k_{x}, k_{y}\right)$, where $E_{\text {kin }}$ is the kinetic energy of electron and $k_{y}$ is the second in-plane component of the wave-vector calculated from the experimental geometry.

For spin-resolved ARPES experiments, the 2D CCD detector is replaced by a spin-detector [Fig. 7.5d]. Different types of such detectors are described in the literature, but mainly so-called classical-Mott and mini-Mott spin-detectors are used [63]. In these devices, the initially spin-polarized beam of electrons, which passed the energy analyzer is accelerated to high energies and then scattered on a heavy-element target. Scattered electrons are then detected by channeltrons, which are placed at particular scattering angles in order to increase the figure-of-merit of 
the detector [chL and chR in Fig. 7.5d]. This allows an effective separation of the spin-polarized electron beam into two channels: spin-up and spin-down electrons. These measurements are performed in the remanence state of the single-domain magnetized sample, and they are usually time consuming due to the fact that backscattered electrons are detected in the spin-detector.

\subsection{Graphene on Lattice-Matched 3d-Metal Surfaces}

Graphene layers (or monolayers of graphite) on lattice matched $3 d$ transition-metal surfaces have been known approximately for three decades. First experiments on graphene/Ni interfaces can be traced back to the mid-1970s [64-68]. In these early experiments segregation of bulk-dissolved carbon on different surfaces of $\mathrm{Ni}$ was investigated in order to explore the influence of the graphene layer on their catalytic activity. Structural stability and different growth modes of graphene layers were studied in these works mainly by means of low-energy electron diffraction (LEED) and Auger-electron spectroscopy. Later on, investigations of the atomic structure of graphene on a Ni surface were performed $[69,70]$. The so-called $f c c-h c p$ arrangement of carbon atoms above the Ni(111) surface was proposed based on the results of surface extended-energy-loss fine-structure spectroscopy studies [69]. The graphene layer on $\mathrm{Ni}(111)$ was found to be flat and slightly expanded compared to bulk graphite (by $\sim 2 \%$ ). The graphene-Ni distance was estimated to be $2.80 \AA$, and the $\mathrm{Ni}(111)$ substrate was found to remain unchanged. The atomic structure of the graphene/Ni(111) system was also investigated by means of LEED intensity analysis of particular diffraction spots [70]. The results of this study did not support the previous conclusions about the system geometry [69], and top-fcc configuration with a slightly buckled graphene layer was found to be the most probable configuration [see Fig. 7.3a for this structure]. This work also predicted a shorter distance of $2.10 \AA$ between the graphene layer and the Ni(111) surface in agreement with the main observations of electron spectroscopy experiments [70].

The electronic structure of the graphene/ $\mathrm{Ni}(111)$ system was investigated by means of angle-integrated photoemission, electron energy-loss spectroscopy, and ionization-loss spectroscopy methods $[69,72]$. The energy bands of graphene were found practically unchanged compared to those of a pure graphite crystal, but an almost rigid shift to higher binding energies of all graphene-derived bands was detected in the experiment, indicating a charge transfer of $0.02 e^{-} /$carbon atom from the $\mathrm{Ni}$ substrate to the graphene overlayer. Further experiments on graphene/Ni(111) $[23,73]$ performed by means of angle-resolved PES showed that the energy shift to higher binding energies is different for $\pi$ - and $\sigma$-derived states indicating the different strength of hybridization between $\mathrm{Ni} 3 d$ valence band states and out-of-plane $\pi$ and in-plane $\sigma$ valence band states of the graphene layer. The orbital hybridization of the valence band states of graphene and the substrate leads to a considerable interaction between $\mathrm{C}$ and $\mathrm{Ni}$ atoms, resulting in a rather short distance between graphene and $\mathrm{Ni}(111)$, and also to the weakening of the $\mathrm{C}-\mathrm{C}$ 


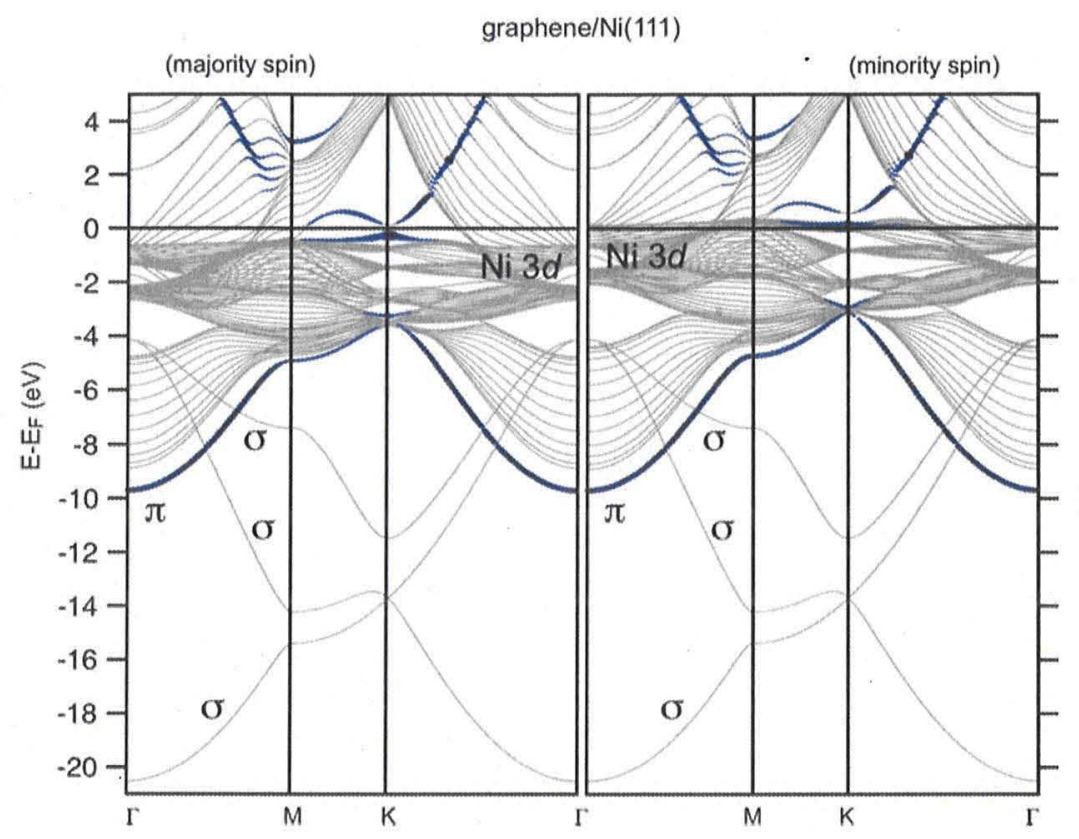

Fig. 7.6 Calculated majority and minority spin band structures for a slab terminated by graphene/ $\mathrm{Ni}(111)$ interface for a most energetically favorable top-fcc configuration. For the blue (darker) lines, the carbon $p_{z}$ character is used as a weighting factor. Data are taken from [71]

in-plane bonds. These results were confirmed by recent experiments $[74,75]$ and theoretical calculations [35, 36, 71, 76] (Fig. 7.6).

This section presents a detailed analysis of the growth and electronic structure of graphene on lattice-matched $3 d$-metal surfaces, focusing mostly on Ni(111). The main experimental observations are presented and discussed in the light of the currently available band-structure calculations.

\subsubsection{Atomic Structure of Graphene Layer on $\mathrm{Ni}(111)$ and $\mathrm{Co}(0001)$}

The STM results presented here were obtained on a graphene layer prepared on thick $\mathrm{Ni}(111)$ grown on $\mathrm{W}(110)[24,75,77-79]$. Fig. 7.7a shows an STM image of single-layer graphene on $\mathrm{Ni}(111)$ after thermal decomposition of propylene. A typical LEED pattern of monolayer graphene on $\mathrm{Ni}(111)$ is presented as an inset. The graphene layer is continuous and exhibits a highly ordered crystallographic structure without any visible defects even over large areas. Since Ni(111) and graphene have the nearly similar lattice parameters (the lattice mismatch is of only $1.3 \%)$, graphene forms a hexagonal $(1 \times 1)$ structure. A higher magnification STM 

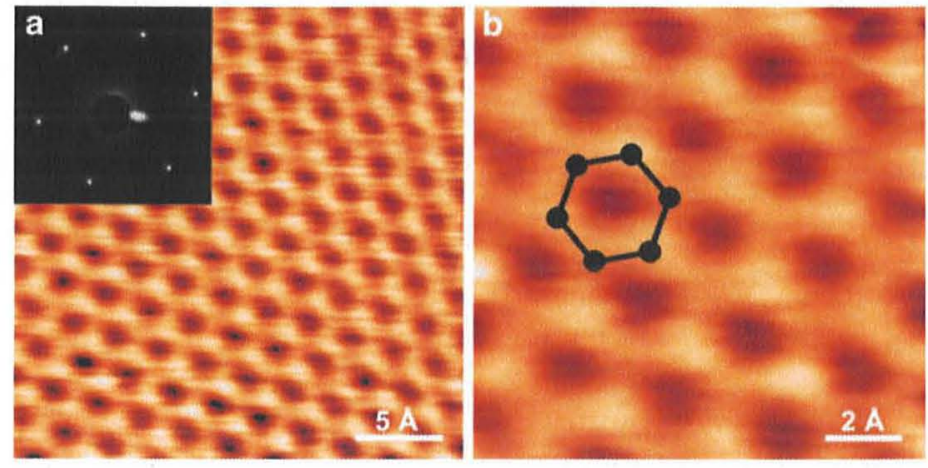

Fig. 7.7 (a) A topographic STM image of the high-quality graphene/Ni(111) system. Tunneling parameters: $U_{\mathrm{T}}=0.002 \mathrm{~V} ; I_{\mathrm{T}}=48 \mathrm{nA}$. The inset shows a LEED image obtained at $63 \mathrm{eV}$. (b) Magnified STM image of the perfect graphene lattice. Tunneling parameters: $U_{\mathrm{T}}=0.002 \mathrm{~V}$; $I_{\mathrm{T}}=48 \mathrm{nA}$ ). The graphene honeycomb is marked in (b). Data are taken from [75]

image of the graphene surface is shown in Fig. 7.7b with the honeycomb of ideal graphene marked in the image.

So far, several possible atomic configurations were considered for the graphene/Ni(111) interface. Three "high-symmetry" structures are known as $h c p-f c c$, top-hcp, and top-fcc. In the top-hcp configuration, the $\mathrm{C}$ atoms are placed directly above the $\mathrm{Ni}$ atoms of the first layer (top site) and the second layer ( $h c p$ site) [76]. In top-fcc, the $\mathrm{C}$ atoms are arranged above the $\mathrm{Ni}$ atoms of the first and third $(f c c)$ layers [Fig. 7.3a]. In the $h c p-f c c$ configuration, the $\mathrm{C}$ atoms are placed above $h c p$ and $f c c$ sites. Three additional configurations were considered recently, which were called bridge-top, bridge-fcc, and bridge-hcp. In these structures, the C atoms are not placed in $h c p-f c c$, top-hcp, and top-fcc sites but in between [80].

At the moment, no clear consensus exists about which of the above described structures is more energetically stable and which kind of structures are observed in experiments. From the theoretical side, Bertoni et al. [76] used density functional theory (DFT) with the Perdew, Burke, and Ernzerhof generalized gradient approximation (GGA-PBE), which indicated the top-fcc to be the most stable atomic configuration at the graphene/Ni(111) interface. DFT-PBE studies were also performed by Kalibaeva et al. [81] reporting that top-fcc structure is the lowest energy configuration, whereas $h c p-f c c$ has been shown to be unstable. The calculations including three additional "low-symmetry" configurations showed that within DTF with GGA-PBE, none of the structures is stable at the experimentally relevant temperatures; with local-density approximation (LDA), the bridge-top configuration was found to be the most energetically favorable one [80]. However, the performed $k$-point sampling of the Brillouin zone performed in this work seems to be not sufficient for this system (compare [80] and [36]), requiring a further investigations of the graphene/Ni(111) system. From the experimental side, Rosei 
et al. [69] and Klink et al. [82] found that the most stable structure is hcp-fcc, whereas Gamo et al. [70] found top- $f c c$ to be the most favorable configuration.

Graphene terraces (Fig. 7.7) have a peak-to-peak roughness of $0.2 \AA$ and show a honeycomb structure with a lattice constant of $2.4 \pm 0.1 \AA$ [Fig. 7.7b], which agrees well with the expected $2.46 \AA$ lattice spacing of graphene $[24,75,77,78]$. STM images show that within each honeycomb carbon atoms corresponding to different sites appear with a different contrast, which can be attributed to the differences in the local stacking of the graphene sheet and the Ni(111) substrate. Therefore, STM images could be interpreted in the following way: Fig. 7.7 shows a single layer graphene, where one half of carbon atoms are situated on top of nickel atoms and the other half occupy one of the two non-equivalent threefold hollow cites - $f c c$ or $h c p$. However, it turns to be impossible to directly identify which of the hollow sites is occupied.

The STM experiments performed on the graphene/Co(0001) system also demonstrate the epitaxial growth in the system with one of the carbon atoms in the graphene unit cell situated above Co surface atom [83]. The position of the second carbon atom is not clear and could be obtained only from the comparison of the calculated band structure with experimental structural and spectroscopy data. The recent calculations for this system predict that top-hcp and top-fcc arrangements at the interface are more favorable with spacing between Co surface and graphene layer of $2.07 \AA$ [83].

\subsubsection{Electronic Structure of Graphene on Lattice-Matched Surfaces}

The lattice matched interface between graphene and $\mathrm{Ni}(111)$ is among the most extensively studied graphene/metal systems [23, 69-71, 73, 75-79, 84-87]. A considerable modification of graphene's electronic structure due to the orbital mixing between Ni $3 d$ and graphene $\pi$ states was predicted theoretically $[35,36,71,76,84]$ and observed recently by NEXAFS spectroscopy [86,87]. Figure 7.8 shows angledependent $\mathrm{C} 1 s$ NEXAFS spectra of graphene on $\mathrm{Ni}(111)$ in comparison with the $\mathrm{C} 1 s$ absorption spectrum of highly oriented pyrolytic graphite (HOPG). The spectrum of HOPG is characterized by the $\mathrm{C} 1 s \rightarrow \pi^{*}$ resonance at $285.3 \mathrm{eV}$ and two $\mathrm{C} 1 s \rightarrow \sigma^{*}$ resonances around $291.6 \mathrm{eV}$ and $292.7 \mathrm{eV}$. The shape of this spectrum can be best described including effects of dynamical screening of the core hole [88]. Comparing the C $1 s$ NEXAFS spectrum of the graphene/Ni(111) system with the reference graphite spectrum, considerable changes in the spectral shapes are observed, indicating a substantial orbital hybridization between graphene and $\mathrm{Ni}$ valence band states at the interface. This is a direct experimental evidence for significant chemical interaction between graphene and $\mathrm{Ni}(111)$, in accordance with the relatively high adsorption energy of $125 \mathrm{meV}$ per $\mathrm{C}$ atom calculated for this system [89]. A new strong feature at $287.1 \mathrm{eV}$ originates from transitions to the 

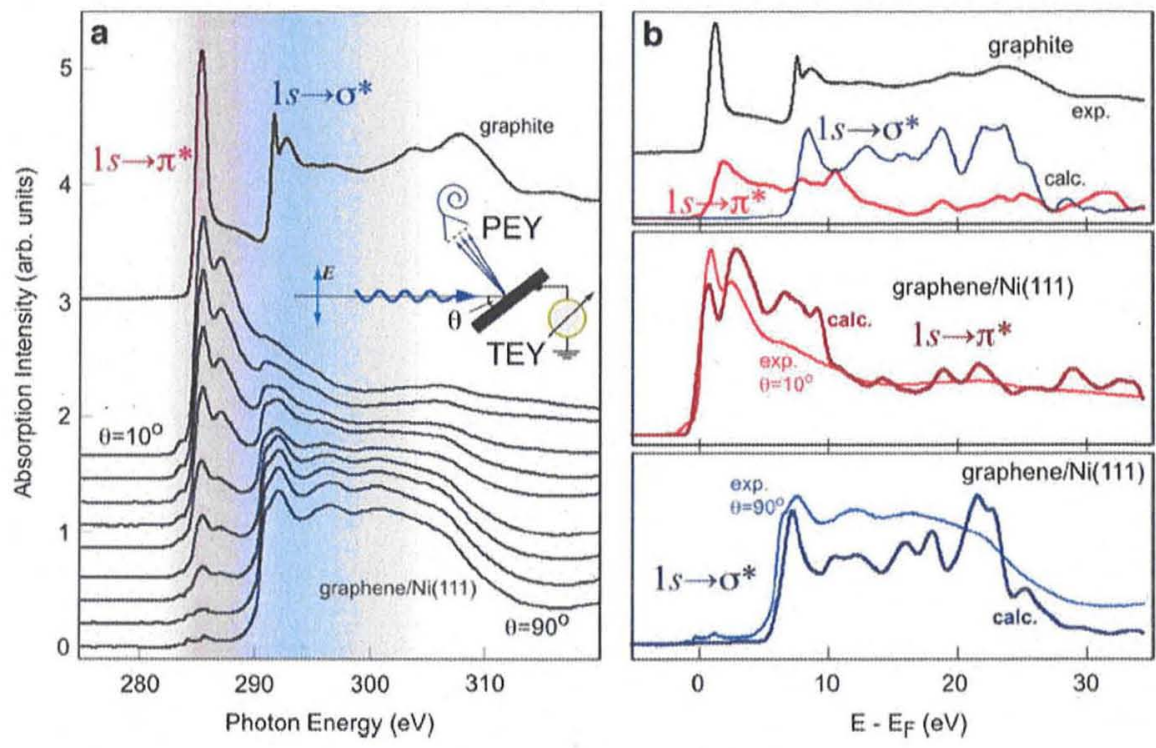

Fig. 7.8 (a) Angle-dependent $\mathrm{C} 1 s$ NEXAFS spectra of the graphene/Ni(111) system measured as a function of angle $\theta$, between polarization vector of the incoming linearly polarized light and the surface normal (see inset for exact experimental geometry). The reference spectrum from HOPG is shown in the upper part of the panel. (b) Comparison between experimental NEXAFS spectra and calculated EELS of graphite and graphene/Ni(111) for two different incident angles, $\theta$, where transitions from C $1 s$ core level on mostly $\pi^{*}$ - or $\sigma^{*}$-states occurred. Experimental data are from $[75,86]$. Theoretically calculated EELS spectra are taken from [76]

states with $\pi$ symmetry, as evident by the angle dependence. The characteristic double-peak structure at $285.5 \mathrm{eV}$ and $287.1 \mathrm{eV}$ can be qualitatively understood already with the ground-state DFT calculations [76], while the dynamics of corehole screening has to be considered for correct reproduction of the spectral profile [87]. In the ground-state approximation, the first $\pi^{*}$ resonance can be roughly associated with the unoccupied C $2 p$ density of states (DOS) located at the $\mathrm{C}$ atoms on top of $\mathrm{Ni}$ atoms, while the second $\pi^{*}$ peak is mainly due to the $\mathrm{C}$ $2 p$ DOS on the $\mathrm{C}$ atoms located at $f c c$ hollow sites $[76,86,87]$. However, in a real experiment individual contributions are considerably mixed and further affected by the core hole and its dynamical screening. The $\sigma^{*}$ resonances are also influenced by the interaction with the substrate: they are visibly broadened and shifted by $0.6 \mathrm{eV}$ to lower energies. The broadening is a result of the increased screening by the substrate electrons. The reduction of the $\pi^{*}-\sigma^{*}$ separation reflects the reduced anisotropy of the potential for outgoing electrons due to the slight ripple in the graphene layer on $\mathrm{Ni}(111)$ accompanied by a softening of the $\mathrm{C}-\mathrm{C}$ bonds.

In the following, we would like to compare our NEXAFS results $[75,86]$ with the recently calculated C $K$-edge EELS spectra for the graphene/ $\mathrm{Ni}(111)$ interface [76]. The calculated EELS spectra are found to agree well with the experimental 
NEXAFS data [see Fig. 7.8b]: (1) the spectra show the same angle (scattering vector) dependence and (2) the experimentally observed NEXAFS features are well reproduced in the calculated EELS spectra. For example, two peaks in the NEXAFS spectra in the $1 s \rightarrow \pi^{*}$ spectral region at $285.5 \mathrm{eV}$ and $287.1 \mathrm{eV}$ photon energy can be assigned to the double peak structure in the calculated EELS spectrum at $0.8 \mathrm{eV}$ and $3.0 \mathrm{eV}$ above the Fermi level [76]. According to the theoretical calculations [76], the first sharp feature in the NEXAFS spectrum is due to the transition of the electron from the $1 \mathrm{~s}$ core level into the interface state $I_{4}$ above the Fermi level (around the $K$ point in the hexagonal Brillouin zone, see Fig. 7.6), which originates from the $\mathrm{C} p_{z}-\mathrm{Ni} 3 d$ hybridization and corresponds to the antibonding orbital between a carbon atom C-top and an interface $\mathrm{Ni}$ atom. The second peak in the NEXAFS spectrum is due to the dipole transition of an electron from the $1 s$ core level into the interface state $I_{5}$ above the Fermi level (around the $M$-point in the hexagonal Brillouin zone, see Fig. 7.6), which originates from $\mathrm{C} p_{z}-\mathrm{Ni} p_{x}, p_{y}, 3 d$ hybridization and corresponds to a bonding orbital between C-top and C- $f c c$ atoms, involving an Ni interface atom. In the case of the NEXAFS C $1 s \rightarrow \sigma^{*}$, the theory also correctly describes the shape of the absorption spectra [76].

Since the $\pi$ electrons of graphene are actively involved in the bonding with the $\mathrm{Ni}$ substrate, the overall strength of the $\mathrm{C}-\mathrm{C}$ bonds is considerably decreased. This fact is reflected in the relatively low growth temperature $\left(500^{\circ} \mathrm{C}-650^{\circ} \mathrm{C}\right.$ depending on the hydrocarbon flux) and high solubility of carbon in $\mathrm{Ni}$. The growth temperature is significantly lower than that used for graphene formation on more passive metal surfaces (e.g., $850^{\circ} \mathrm{C}-1,000^{\circ} \mathrm{C}$ in the case of graphene/Pt or graphene/Ir), where the $\mathrm{C}-\mathrm{C}$ bonds are not weakened so drastically by the substrate. The metastable equilibrium state of graphene on $\mathrm{Ni}(111)$ associated with the permanent formation and re-evaporation of graphene flakes can be reached in the temperature window as low as $650^{\circ} \mathrm{C}-670^{\circ} \mathrm{C}$ [79] [Fig. 7.9a, b]. CVD growth is assisted by carbon dissolving in $\mathrm{Ni}$ and segregation to the surface, as can be judged from direct monitoring of the growth process with high-resolution $\mathrm{C} 1 s$ PES. Figure $7.9 \mathrm{~b}$ shows the $\mathrm{C} 1 s$ PE spectra of the final graphene film grown on $\mathrm{Ni}(111)$ obtained at different substrate temperatures [79]. In all cases, the main peak at $284.7 \mathrm{eV}$ is asymmetric and can be reliably fitted using a single component with Doniach-Sunjic lineshape. The fit parameters of this peak are comparable with those reported for the C $1 \mathrm{~s}$ line in other graphene/TM systems [41]. The small feature observed at around $283 \mathrm{eV}$ is characteristic of carbon atoms with reduced coordination (as compared to graphene): atomic carbon dissolved in $\mathrm{Ni}$ and, possibly, carbon atoms at the borders of graphene flakes. Similar features were observed upon dissociation of $\mathrm{CO}$ on $\mathrm{Rh}$ nanostructures and were associated with atomic carbon [90,91]. Since this component results from a disordered phase, its appearance is undesirable. From Fig. 7.9, it is evident that with increasing temperature the signal at $283 \mathrm{eV}$ gradually decreases and vanishes completely close to the equilibrium growth conditions. This implies that all atomic carbon from the substrate is activated at this temperature and participates in graphene formation and/or the contribution from the borders of graphene flakes is reduced due to the reduced nucleation density. 


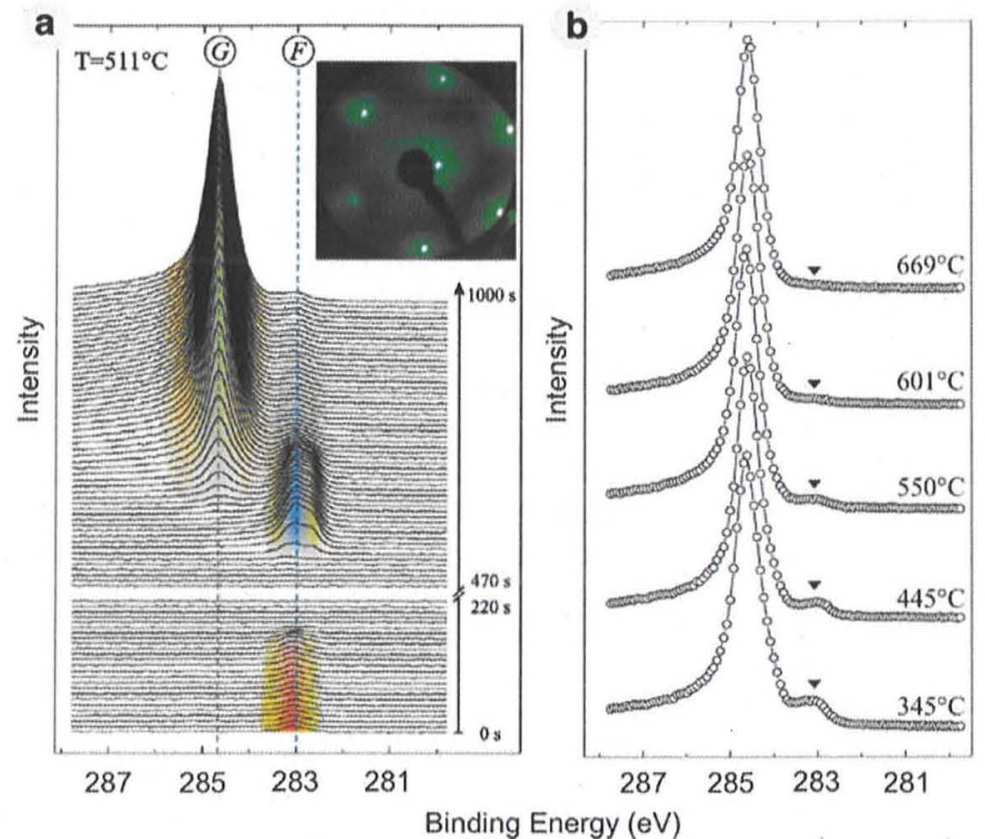

Fig. 7.9 (a) Time evolution of the PES intensity in the $\mathrm{C} 1 s$ region during the graphene growth. Individual spectra were recorded every 10 s. $F$ and $G$ mark the signals from $\mathrm{C}_{3} \mathrm{H}_{6}$ fragments and graphene, respectively. The inset shows a LEED pattern (primary energy was $80 \mathrm{eV}$ ) after growth of graphene. (b) $\mathrm{C} 1 s$ PES spectra of the resulting graphene layer obtained at different temperatures. Data are taken from [79]

Figure 7.10 shows two series of ARPES spectra measured along the $\Gamma-K$ direction of the hexagonal Brillouin zone of the graphene/Ni(111) system [75]. Each series is extracted from the 3D sets of data of photoemission intensity $I\left(E_{\mathrm{B}}, k_{x}, k_{y}\right)$, where $E_{\mathrm{B}}$ is the binding energy and $k_{x}, k_{y}$ are the orthogonal components of the inplane wave vector. The presented photoemission data are in very good agreement with the previously published results $[22,23,73,74,78]$. In Fig. 7.10, one can clearly discriminate dispersions of the graphene $\pi$ - and $\sigma$-derived states in the region below $2 \mathrm{eV}$ of the binding energy (BE) as well as $\mathrm{Ni} 3 d$-derived states near $E_{\mathrm{F}}$. The binding energy of the graphene $\pi$ states in the center of the Brillouin zone (in the $\Gamma$ point) equals to $10.1 \mathrm{eV}$, which is approximately by $2.4 \mathrm{eV}$ larger than the binding energy of these states in pure graphite [77,86]. The shift to larger binding energy is different for $\sigma$ and $\pi$ valence band graphene-derived states. This behavior can be explained by the different hybridization strength between these states and $\mathrm{Ni} 3 d$ valence band states, which is larger for the out-of-plane oriented $\pi$ states compared with the one for the in-plane oriented $\sigma$ states of the graphene layer. The effect of hybridization between $\mathrm{Ni} 3 d$ and graphene $\pi$ states can be clearly demonstrated in the region around the $K$ point of the Brillouin zone: (1) one of the $\mathrm{Ni} 3 d$ bands at $1.5 \mathrm{eV}$ 

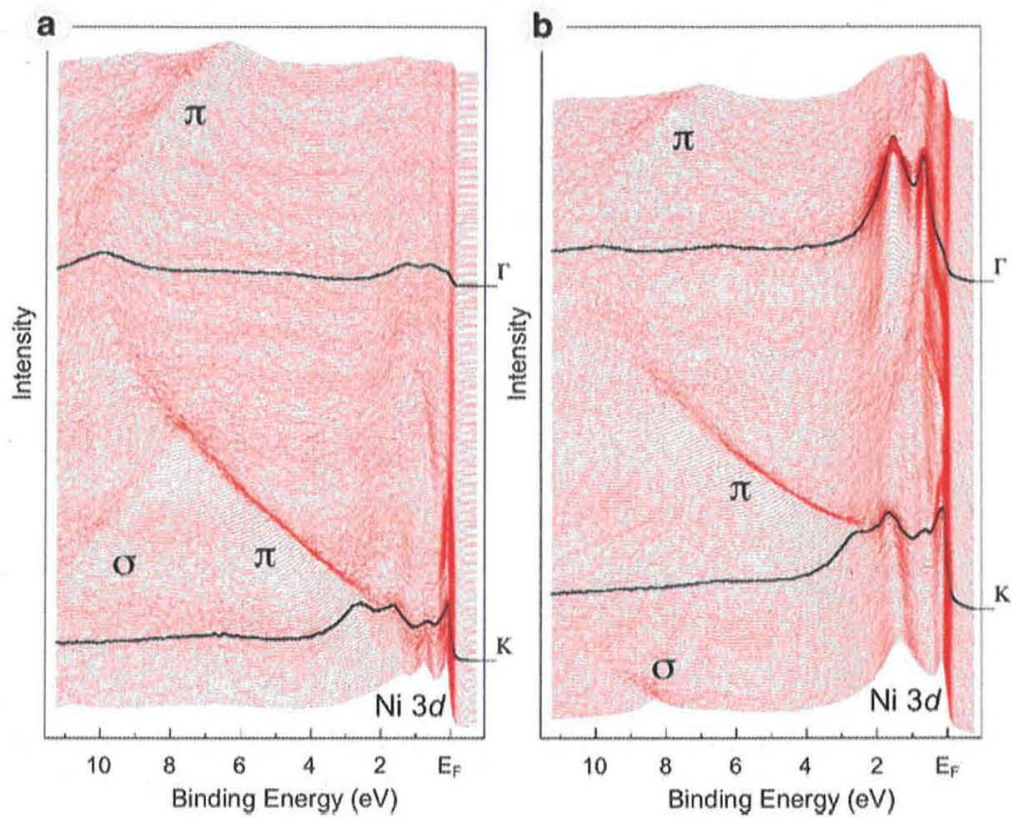

Fig. 7.10 Angle-resolved photoemission spectra of the graphene/Ni(111) system recorded along the $\Gamma-K$ direction of the hexagonal Brillouin zone at (a) $70 \mathrm{eV}$ and (b) $100 \mathrm{eV}$ of photon energy. Spectra corresponding to $\Gamma$ and $K$ points are marked by thick black lines. Data are taken from [75]

of $\mathrm{BE}$ changes its binding energy by $\approx 150 \mathrm{meV}$ to larger $\mathrm{BE}$ when approaching the $K$ point; (2) a hybridization shoulder is visible in photoemission spectra, which disperses from approximately $1.6 \mathrm{eV}$ to the binding energy of the graphene $\pi$ states at the $K$ point. The strong hybridization observed in PES spectra underlines the fact that the $\pi$ states might become spin-polarized and might gain a non-zero magnetic moment due to the admixture of the Ni $3 d$ states into the $\mathrm{C} \pi$ states.

Considering the electronic band structure of the graphene/Ni(111), the region around the $K$ point delivers the most interesting and important information with respect to the possible spin-filtering effects in the graphene/FM or FM/graphene/FM sandwich-like structures. First, the spectral function of the graphene layer on $\mathrm{Ni}(111)$ is characterized by the absence of well-ordered structure of the graphene $\pi$-bands in the vicinity of the Fermi level and second, the Dirac-cone is not preserved. Both observations can be attributed to a strong interaction between graphene layer and metallic substrate leading to a strong hybridization between the graphene $\pi$ and the Ni $3 d$ valence band states. In the vicinity of the $K$ point, a number of photoemission peaks can be clearly distinguished: (1) a sharp peak about the Fermi level at $0.1-0.2 \mathrm{eV} \mathrm{BE,} \mathrm{(2)} \mathrm{a} \mathrm{graphene} \pi$-states-related peak at $2.65 \mathrm{eV}$ $\mathrm{BE}$, (3) two peaks at $0.7 \mathrm{eV}$ and $1.65 \mathrm{eV} \mathrm{BE}$.

The analysis of the experimentally obtained electronic structure has recently been performed in [75] relying mainly on two comprehensive sets of electronic 
structure calculations currently available for the graphene/ $\mathrm{Ni}(111)$ system $[36,76]$. Calculations by Bertoni et al. [76] predict the existence of three interface states below the Fermi level originating from the strong hybridization between the $\mathrm{Ni}$ $3 d$ and the graphene $\pi$ states and corresponding to: $\left(I_{1}\right)$ bonding between $\mathrm{C}-f c c$ and interface Ni atoms; $\left(I_{2}\right)$ bonding between C-top and interface Ni atoms; $\left(I_{3}\right)$ antibonding between $\mathrm{C}-f c c$ and interface Ni atoms. Karpan et al. [36] performed the band structure calculations of the graphene/Ni(111) system with the major emphasis on the investigation of the spin-dependent transport properties of the $\mathrm{Ni}$ /graphene/Ni sandwich structures. Both calculations yielded a quite complicated band structure of the graphene/Ni(111) system around $E_{\mathrm{F}}$ due to the strong hybridization between the graphene and the Ni valence band states.

The interpretation of the experimentally observed photoemission features around the Fermi level could be performed as presented in Ref. [75]. The photoemission peak close to $E_{\mathrm{F}}(0.1-0.2 \mathrm{eV} \mathrm{BE})$ could be considered as a combination of the interface state $I_{3}$ (both spins) with a large contribution of the graphene $\pi$-character and the Ni $3 d(\downarrow)$-band. The second peak at $0.7 \mathrm{eV} \mathrm{BE}$ could be assigned to the combination of the Ni $3 d(\uparrow)$ - and Ni $3 d(\downarrow)$-bands present in both calculations. The feature at $1.65 \mathrm{eV}$ could be considered as a combination of $\mathrm{Ni} 3 d(\uparrow)$-band and $I_{2}(\downarrow)$-state with a large graphene $\pi$-character. The last photoemission peak $(2.65 \mathrm{eV}$ BE) could be assigned to the interface state $I_{2}(\uparrow)$ with large contribution of the graphene $\pi$-character.

In order to check the theoretical predictions concerning the CPP spin-dependent electronic transport properties of the ideal graphene/ $\mathrm{Ni}(111)$ interface, an analysis of the constant energy photoemission maps close to $E_{\mathrm{F}}$ was performed in [75] (Fig. 7.11). However, flat Ni $3 d$ bands, which dominate the photoemission intensity around $E_{\mathrm{F}}$ make the detailed analysis rather difficult. The energy cuts were taken at
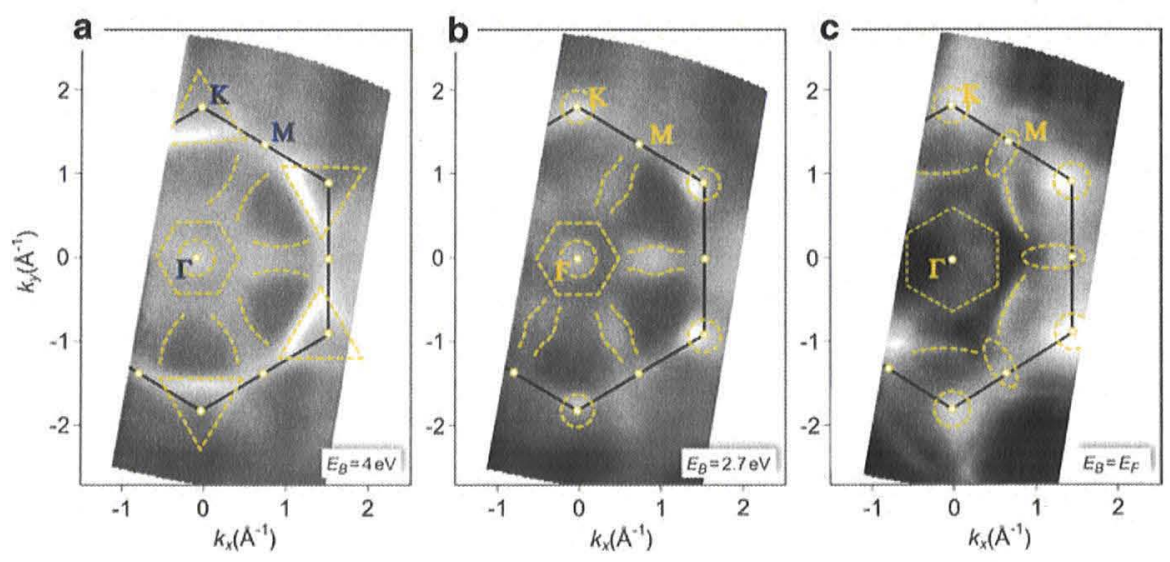

Fig. 7.11 Constant energy cuts of the 3D data sets in the energy-wave vector space, $I\left(E_{\mathrm{B}}, k_{x}, k_{y}\right)$, obtained through a $\beta$-scan from the graphene/ $\mathrm{Ni}(111)$ system at $100 \mathrm{eV}$ of photon energy. The energy cuts are taken at (a) $4 \mathrm{eV}$ and (b) $2.7 \mathrm{eV}$ BE as well as at (c) $E_{\mathrm{F}}$. Data are taken from [75] 
(a) $4 \mathrm{eV}$ and (b) $2.7 \mathrm{eV}$ of $\mathrm{BE}$ as well as at (c) the Fermi level. The energy cut at $E_{\mathrm{B}}=4 \mathrm{eV}$ shows characteristic photoemission intensity patterns of the graphene layer, which reflect the symmetry of the system. Below the Dirac point (crossing of straight dispersion lines of $\pi$ states in free-standing graphene), the graphene $\pi$ bands are visible in the first Brillouin zone, whereas no bands can be seen in the second one [92]. Additionally, several energy bands are present in the middle of the Brillouin zone (outlined by dashed yellow lines), which also show hexagonal symmetry. These bands originate from the hybridization of the $\mathrm{Ni}$ and graphene valence band states. The constant energy cut taken in the region of the minimal binding energy of the graphene $\pi$ states $\left(E_{\mathrm{B}}=2.7 \mathrm{eV}\right)$ is shown in Fig. 7.11b. In the case of graphene/Ni(111), the Dirac point is not preserved due to the strong hybridization of Ni $3 d$ and graphene $\pi$ states around the $K$ point. This can also be directly recognized at this energy cut, where graphene $\pi$ states produce broad intensity spots instead of sharp points in the wave-vector space. As in the previous case, we observe a number of valence band states in the middle part of the Brillouin zone, which again could be assigned to the hybridization-derived states.

The most interesting and important information in view on the spin-dependent transport properties of the graphene/ $\mathrm{Ni}(111)$ system can be extracted from the constant energy cut obtained at the Fermi energy, which is presented at Fig. 7.11c. Already the analysis of Fig. $7.10 \mathrm{a}, \mathrm{b}$ shows that the photoemission intensity is increased around the $K$ point and along the $K-M$ direction of the hexagonal Brillouin zone, that correlates with the increased photoemission intensity observed in the energy cut shown in Fig. 7.11c for the Fermi energy. Additionally, a number of arcs surrounding the $K$ points and weak (but distinguished) diamond-shape regions of increased intensity are clearly visible in the middle part and around the $M$ points of the Brillouin zone, respectively. Upon the comparison of the obtained photoemission results for the graphene/Ni(111) system (Figs. 7.10 and 7.11) as well as the Fermi-energy cut with the band structure calculations for this system [35, 36, 71, 76] (Fig. 7.6), we find very good agreement between theory and experiment. Particularly, the region around the Fermi level for the ideal graphene/ $\mathrm{Ni}(111)$ system is well reproduced in the experiment, confirming the main predictions of the theory. However, more experimental and theoretical efforts are required to determine the spin character of individual bands.

\subsubsection{Magnetism of Graphene on the Ni(111) Surface}

The earlier discussed hybridization between graphene $\pi$ and Ni $3 d$ valence band states leads also to a partial charge transfer of the spin-polarized electrons from $\mathrm{Ni}$ to $\mathrm{C}$. This leads to the appearance of induced effective magnetic moment of carbon atoms, which can be detected in experiments sensitive to the magnetic state of particular elements, such as XMCD. Figure 7.12 shows XMCD spectra of the graphene/ $\mathrm{Ni}(111)$ system collected at the $\mathrm{Ni} L_{2,3}$ (left panel) and $\mathrm{C} K$ (right panel) absorption edges in the TEY and PEY modes, respectively [86]. 


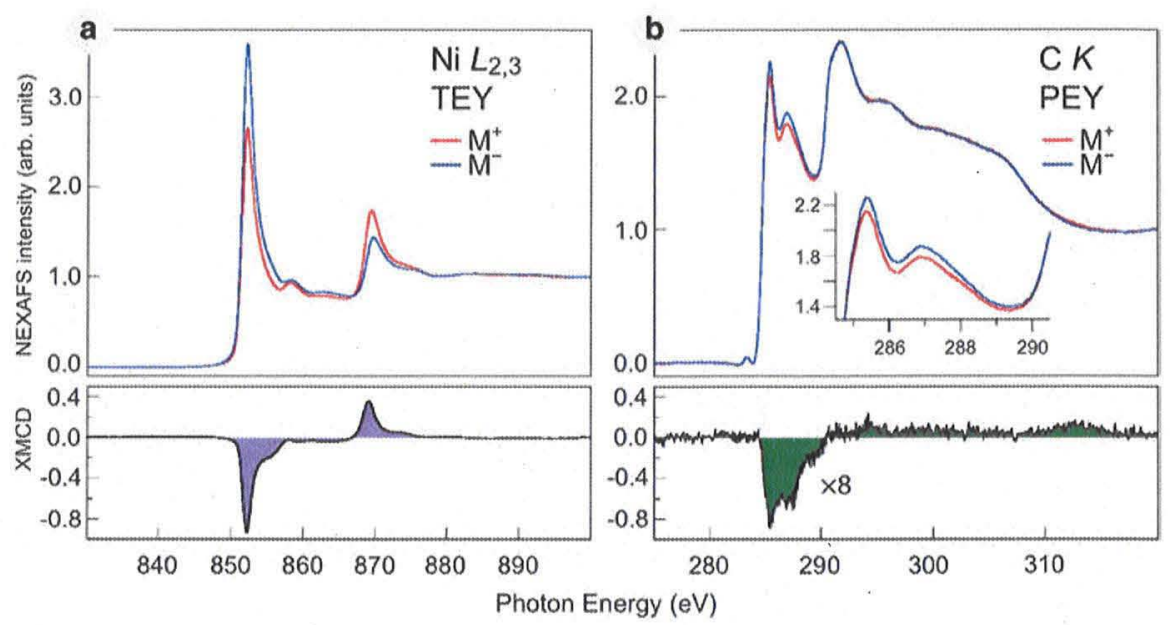

Fig. 7.12 XMCD spectra of the graphene/Ni(111) system measured at the Ni $L_{2,3}$ (a) and C $K$ absorption edges (b). The corresponding differences reflecting the strength of the dichroic signal are shown in the lower part of the respective figures. Data are taken from $[75,86]$

The Ni $L_{2,3}$ XMCD spectrum (white line as well as fine structure behind the absorption edge) is in perfect agreement with the previously published spectroscopic data [93-95] with the extracted values of spin- and orbital-magnetic moments $\mu_{S}=0.69 \mu_{B}$ and $\mu_{L}=0.07 \mu_{B}$, respectively, which are in very good agreement with the previously published experimental ones $[93,96]$. The experimentally obtained value of the spin-magnetic moment is in agreement with the theoretically calculated one $\mu_{S}=0.67 \mu_{B}$ for the graphene/Ni(111) system [76]. For the most energetically favorable configuration of carbon atoms on Ni(111), C-top-C-fcc, calculations predict a reduction of the spin-magnetic moments of $\mathrm{Ni}$ interface atoms by $16 \%$ till $0.56 \mu_{B}$. Experimental data collected in the PEY mode (more surface/interface sensitive) at the Ni $L_{2,3}$ absorption edge also demonstrate slight reduction of spin moment of surface $\mathrm{Ni}$ atoms till $\mu_{S}=0.63 \mu_{B}[75,86]$ that is consistent with theoretical calculations [76].

The most important and interesting result of these XMCD experiments on the graphene/ $\mathrm{Ni}(111)$ system is the observation of the relatively large dichroic contrast at the C $K$ absorption edge [Fig. 7.12b]. The C $K$ XMCD spectrum reveals that the major magnetic response stems from transitions of the $1 s$ electron onto the $\pi^{*}$-states, while transitions onto the $\sigma^{*}$-states yield very weak (if any) magnetic signal indicating that only the $\mathrm{C} 2 p_{z}$ orbitals of the graphene layer are magnetically polarized due to the mixing with the $\mathrm{Ni} 3 d$ band. As discussed earlier, the sharp structure at the $1 s \rightarrow \pi^{*}$ absorption edge originates from hybridized C $p_{z}-\mathrm{Ni} 3 d$ and $\mathrm{C} p_{z}-\mathrm{Ni} p_{x}, p_{y}, 3 d$ states (see earlier discussion and [76]).

At the C $K$ absorption edge, the electron transitions occur from the non-spinorbit split $1 s$ initial states to the $2 p$ final states and thus, in the analysis of the dichroism effect at the $K$ edge one equation in the "sum" rules is missed. This 
means that the XMCD signal at $K$ edges provides the information only on the orbital magnetic moment $\mu_{\text {orb }}[58,59,97]$. In $[75,86]$, the value of the $C$ orbital magnetic moment was estimated to be $\mu_{\text {orb }}=1.8 \pm 0.6 \times 10^{-3} \mu_{B}$. Comparison of the presented results from $[75,86]$ with recent experimental studies of induced magnetic effects in nonmagnetic materials $[98,99]$ allows to estimate a value of spin magnetic moment of carbon atoms in graphene/ $\mathrm{Ni}(111)$ in the range $0.05-$ $-0.1 \mu_{B}[75,86]$. An analysis of the calculated spin-resolved electronic structure of graphene/ $\mathrm{Ni}(111)$ [76] is difficult due to complicated behavior of the majority and minority spin interface states. In [76], the calculated magnetic moment is very small: $-0.01 \mu_{B}$ and $0.02 \mu_{B}$ for C-top and C- $f c c$, respectively, although the magnetic splitting of the majority and minority parts of the interface states $I_{3}$ and $I_{4}$ was found between 0.13 and $0.55 \mathrm{eV}$, respectively.

The experimentally observed effective magnetic moment of carbon atoms of the graphene layer on $\mathrm{Ni}(111)$ is also confirmed by our spin-resolved photoemission study [75]. The main results are presented in Fig. 7.13 showing (a) the spin-resolved spectra and (b) the corresponding spin polarization as a function of the binding energy of $\mathrm{Ni}(111)$ and the graphene/ $\mathrm{Ni}(111)$ system measured in the region of $\mathrm{Ni} 3 d$ valence band with a photon energy of $65 \mathrm{eV}$ at room temperature in normal emission geometry. The formation of graphene on $\mathrm{Ni}(111)$ strongly modifies the valence band spectrum of $\mathrm{Ni} 3 d$ as a result of the strong interaction between valence band states of graphene and Ni. These considerable modifications of the spin-resolved structure of $\mathrm{Ni} 3 d$ states as well as a reduction of spin polarization at the Fermi level could
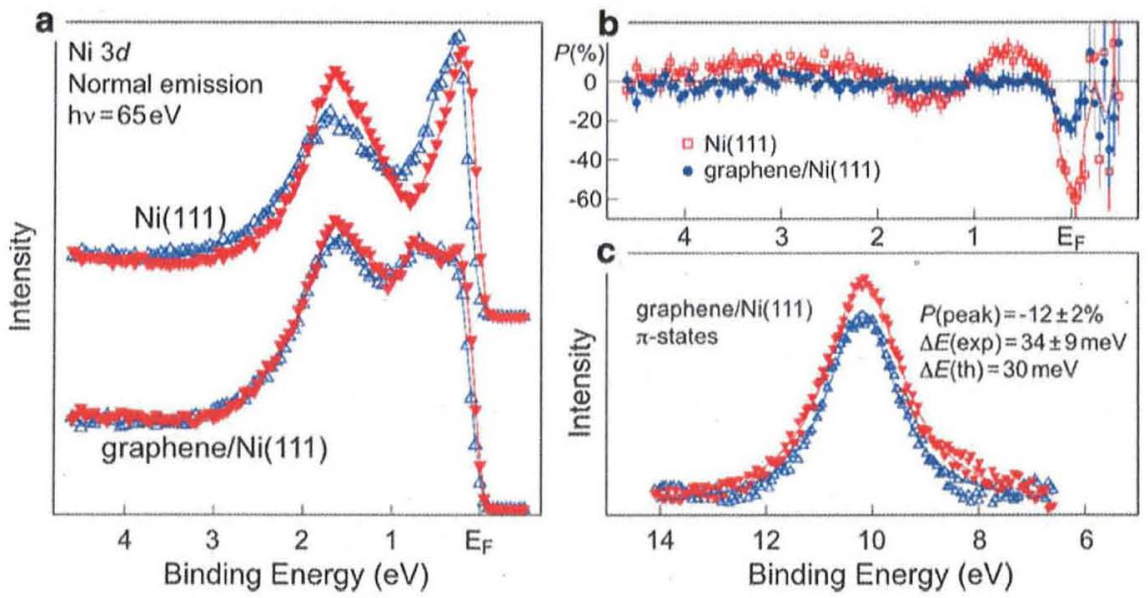

Fig. 7.13 (a) Spin-resolved PES spectra and (b) corresponding spin polarization of the Ni $3 d$ valence band states as a function of the binding energy for $\mathrm{Ni}(111)$ and the graphene/Ni(111) system. (c) Spin-resolved photoemission spectra of the $\pi$-states of graphene on $\mathrm{Ni}(111)$. All spectra were collected in the normal emission geometry with the photon energy of $65 \mathrm{eV}$. In (a) and (c), open and filled triangles denote spin-up and spin-down electron channels, respectively. Data are taken from [75] 
be taken as an indication of the decreasing of magnetic moment of $\mathrm{Ni}$ atoms at the interface between graphene and $\mathrm{Ni}(111)$, which was predicted by theory [76].

The spin-resolved spectrum of the graphene $\pi$ states of graphene/ $\mathrm{Ni}(111)$ measured in normal emission geometry with photon energy of $65 \mathrm{eV}$ is shown in Fig. 7.13c. These results demonstrate a clear spin contrast for the graphene $\pi$ states with the maximum spin polarization of about $(-12 \pm 2 \%)$ and the exchange splitting of about $34 \pm 9 \mathrm{meV}$, which is in rather good agreement with the value extracted from theoretical works [76, 100]. A comparison of these spin-resolved spectra including their sign with the background spin polarization originating from the Ni $3 d$ states give an opportunity to conclude that the spin moment of graphene is aligned antiparallel to the magnetization of $\mathrm{Ni}$ implying an antiparallel magnetic coupling of graphene to Ni. However, this conclusion is based only on the normal emission spin-resolved measurements and has to be clarified by more intensive spin-resolved PES experiments. Here, we would like to note that the experimental evidence of a pronounced spin polarization at room temperature together with the splitting of the $\pi$ states in the graphene/ $\mathrm{Ni}(111)$ system are in contradiction to the results previously reported by Rader et al. [101], where the absence or very small spin polarization of the graphene $\pi$ states was observed. The origin of this discrepancy is, however, not clear at the moment and further spin-polarized PES investigations of the graphene/ $\mathrm{Ni}(111)$ system should be undertaken in order to resolve this issue.

\subsection{Graphene on Lattice-Mismatched $4 d, 5 d$-Metal Surfaces}

As shown in the previous section, in the case of close lattice parameters between graphene and hexagonally close-packed metal surface, lattice-matched structures are formed, like for graphene on $\mathrm{Co}(0001)$ [83] and $\mathrm{Ni}(111)$ [53]. However, larger mismatches lead to the formation of superstructures with large periodicity, which were observed on $\mathrm{Pt}(111)$ [8, 41, 102-108], $\operatorname{Ir}(111)$ [40, 41, 109-114], $\operatorname{Pd}(111)$ [41, 115], $\operatorname{Rh}(111)[33,41,51,116,117]$, or $\operatorname{Ru}(0001)$ [41-44,46, 116, 118-121]. Already more than four decades ago, graphene layers were stabilized on noble metal surfaces upon high temperature annealing of Pt and Ru single crystals [102, 103, 116]. After hydrocarbon decomposition or carbon surface segregation on metal surfaces at elevated temperatures sharp satellite spots or segmented rings were observed in LEED [102-104, 109, 116, 119, 122], which were attributed to the formation of graphene layers. However, an extended temperature treatment during the segregation process may lead not only to single layer, but also to multilayers of graphene or even to bulk graphite formation. In the subsequent studies [22, 73, 112, 123, 124], single-layer graphene formation could be discriminated by its characteristic band structure in ARPES (see also previous section). In this section, we will focus on the atomic structure of single-layer graphene on $\operatorname{Ir}(111), \operatorname{Ru}(0001)$ and $\operatorname{Rh}(111)$ surface, as well as on the changes of electronic structure of graphene associated with the bonding to these substrates. 


\subsubsection{Structure of Graphene on $\operatorname{Ir}(111), R u(0001)$, and $R h(111)$}

Several growth methods were reported for the preparation of highly ordered graphene monolayers on $\operatorname{Ir}(111)$, including pyrolysis of preadsorbed ethylene and graphene growth at elevated temperature, which is also referred to as temperature programmed growth (TPG) and low-pressure CVD of ethylene on hot Ir surface [40, $110,126]$. Upon using high growth temperatures up to $1,320 \mathrm{~K}$, the latter technique allows the preparation of single-layer graphene domains of unprecedented quality. Graphene flakes prepared by this method are dislocation-free and fully coherent over step edges reaching at least micrometers in size [40, 110, 126]. The STM topographic image in Fig. 7.14a shows the perfectly ordered moiré superstructure of single-layer graphene on $\operatorname{Ir}(111)$ prepared by high-temperature decomposition of propene [125]. The moiré superstructure was reported to have a periodicity of $25.3 \pm 0.4 \AA[40,110,126,127]$, which corresponds to 9.32 Ir lattice constants showing that the structure is incommensurate. Depending on tunneling voltage, the moiré superstructure contrast changes considerably indicating a strong influence

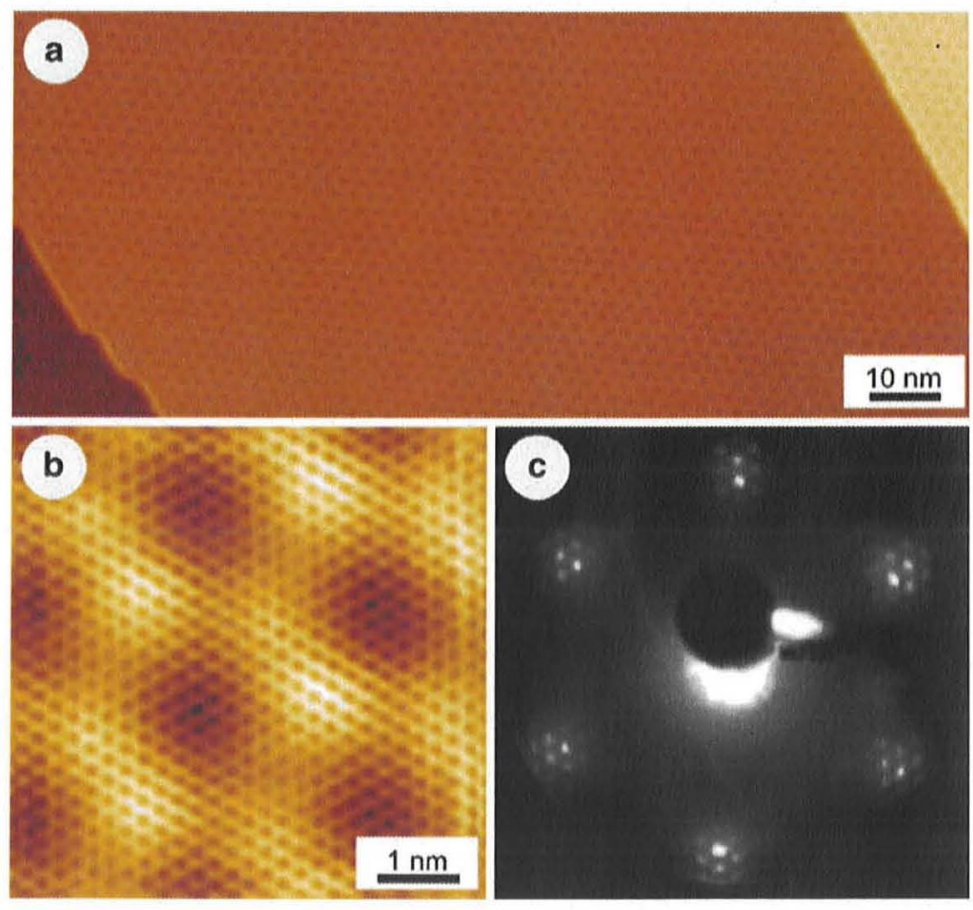

Fig. 7.14 (a) Large-scale STM image of an epitaxial graphene layer on the $\operatorname{Ir}(111)$ surface. (b) Atomically resolved STM image of the moiré structure of the graphene layer on $\operatorname{Ir}(111)$. (c) LEED image of the graphene layer on $\operatorname{Ir}(111)$ taken at a primary electron energy of $70 \mathrm{eV}$. Tunneling parameters for STM images: (a) $U_{\mathrm{T}}=1.12 \mathrm{~V} ; I_{\mathrm{T}}=0.18 \mathrm{nA}$; (b) $U_{\mathrm{T}}=0.01 \mathrm{~V} ; I_{\mathrm{T}}=2.56 \mathrm{nA}$. Data are taken from [125] 
of the electronic structure in STM $[40,110,126,127]$. The high-resolution STM image in Fig. 7.14b shows no atomic-scale defects, confirming a high crystalline quality of single layer graphene on the $\operatorname{Ir}(111)$ surface [125]. The full graphene honeycomb lattice is clearly visible in all areas of the moiré supercell. The LEED pattern of the graphene/ $\operatorname{Ir}(111)$ surface is shown in Fig. 7.14c [125]. The pattern shows hexagonal symmetry consisting of a six pairs of iridium and carbon spots surrounded by hexagonally arranged satellites correspond to the superstructure. The LEED measurements yield a value of $25.8 \pm 2.0 \AA[40,110,126,127]$ for the periodicity of the superstructure, which coincides with the value extracted from the STM images. Beyond the most abundant graphene moiré structure on $\operatorname{Ir}(111)$ described above additional orientations of graphene were reported recently [111]. A combined low-energy electron microscopy (LEEM), selected-area LEED and STM investigation revealed three additional moiré domains, which are rotated by $\sim 14^{\circ}, \sim 18.5^{\circ}$, and $\sim 30^{\circ}$ with respect to the most abundant orientation. Along with rotational variants, liner defect structures in graphene sheets were observed by LEEM [111]. STM shows that these defects are wrinkles in the graphene layer, suggesting that the graphene sheets delaminate locally upon substrate contraction during cooling after growth at higher temperatures $[111,128]$. Upon temperature cycling, a hysteresis in the appearance and disappearance of the wrinkles was observed [128]. However, for possible applications a controlled graphene growth is highly desirable allowing fabrication of large defect-free well-oriented monolayer graphene sheets. Recent studies show that millimeter-sized graphene films of a single orientation and unprecedented structural perfection can be prepared on $\operatorname{Ir}(111)$ by a cyclic growth exploiting the different growth and oxygen etching speeds of the domain variants [129].

On the $\mathrm{Ru}(0001)$ surface graphene also forms a moiré structure. A topographic STM image presented in Fig. 7.15a shows large graphene flakes formed on the terraces of the $\mathrm{Ru}(0001)$ surface after the carbon segregation at high temperature [42]. Figure $7.15 b$ shows atomically resolved STM image of graphene on $\mathrm{Ru}(0001)$ indicating a strong atomic contrast variation within the supercell of the moire structure. Contrary to the STM data obtained on graphene/Ir(111), here the full graphene honeycomb lattice is only visible at the moiré maxima $[9,42]$. At the two nonequivalent minima of the moiré supercell only one of the two carbon sublattices is resolved. The observed atomic contrast across the graphene supercell is assigned to the local variation of the interaction strength between the graphene sheet and the underlying ruthenium surface depending on the atomic registry of carbon atoms with the $\mathrm{Ru}$ atoms. The two nonequivalent minima in the graphene unit cell differ with respect to the $\mathrm{Ru}$ atoms in the substrate layer. In one region, the carbon atoms occupy top-hcp sites, in the other top-fcc sites. Recent surface X-ray diffraction (SXRD) investigation [43] showed that contrary to the previously proposed periodicity of approximately $(11 \times 11)$, i.e., with $(12 \times 12)$ graphene unit cells overlaid, the true structure is a $(25 \times 25)$ graphene on $(23 \times 23) \mathrm{Ru}$ structure, meaning that the unit cell consists of four moiré subcells. More recently, the graphene/Ru(0001) structure was further refined upon a detailed analysis of the SXRD data [130]. The proposed structure shows an excellent agreement with previous STM studies $[9,42]$. 

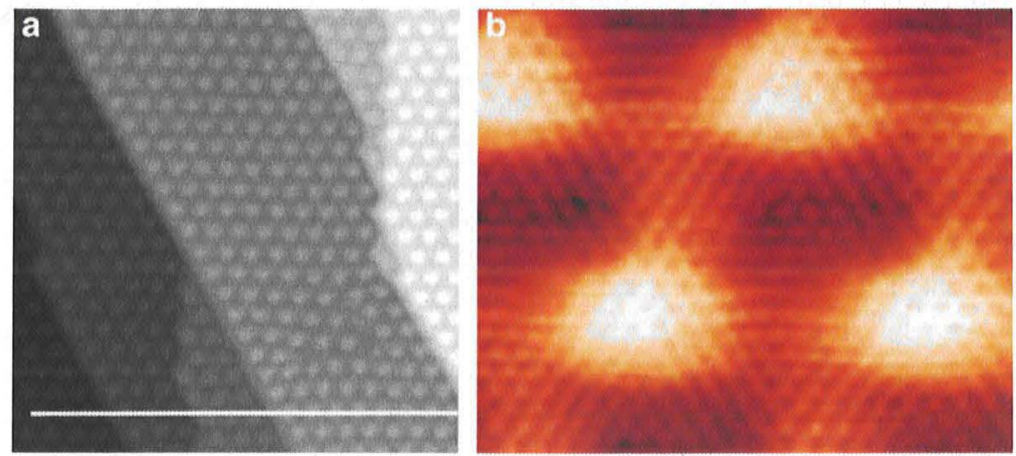

Fig. 7.15 (a) $500 \AA \times 500 \AA$ topographic STM image recorded after annealing Ru(0001) sample at $1,470 \mathrm{~K}$. The surface is fully covered by graphene and Ru step edges are aligned along the main directions of the overlayer, indicating a restructuring of the underlying Ru surface. (b) $50 \AA \times 50 \AA$ atomically resolved image of the graphene overlayer on $\mathrm{Ru}(0001)$. The image shows three different levels of apparent heights, namely four bright maxima, a dark minimum in the center between the three maxima on the right hand side, and a less dark minimum between the three maxima on the left hand side. Tunneling parameters for STM images: (a) $I_{\mathrm{T}}=1 \mathrm{nA}, U_{\mathrm{T}}=-0.2 \mathrm{~V}$; (b) $I_{\mathrm{T}}=1 \mathrm{nA}$, $U_{\mathrm{T}}=-0.05 \mathrm{~V}$. Data are taken from [42]

A pronounced corrugation of both graphene and ruthenium substrate at the interface was found supporting the idea of a chemisorbed graphene layer with significant interaction with the substrate. Moreover, areal chirality in the in-plane movements in graphene layer on $\mathrm{Ru}(0001)$ was observed [130].

In the following, we would like to focus on the graphene/ $\mathrm{Rh}(111)$ system exhibiting a rather complicated moiré pattern. An overview of a graphene layer on the $\mathrm{Rh}(111)$ surface is shown in Fig. 7.16a [117]. The graphene domain exhibits a highly ordered moiré structure without any visible defects even over large areas. $\mathrm{Rh}$ steps are aligned along the main crystallographic directions of the graphene moiré, which is attributed to the substantial reshaping of the $\mathrm{Rh}(111)$ surface during the graphene growth process in order to accommodate the periodicity and orientation of the graphene overlayer. The apparent vertical corrugation of the graphene monolayer is measured to be in the range of $0.5-1.5 \AA$ (peak-to-peak) depending on the tunneling conditions [see inset in Fig. 7.16a]. LEED pattern of the graphene moiré on $\mathrm{Rh}(111)$ is presented in Fig. 7.16d. Qualitative analysis of STM and LEED images show that the close-packed directions of graphene and the unit cell vectors of the moire are parallel to the close-packed $\langle 1 \overline{1} 0\rangle$ directions of $\mathrm{Rh}(111)$. From the LEED images, a periodicity of $2.90 \pm 0.1 \mathrm{~nm}$ of the moiré superstructure on $\mathrm{Rh}(111)$ was calculated, which is in reasonable agreement with the average distance between the neighboring moiré features measured in STM images and corresponds roughly to 12 times the lattice constant of graphene and 11 times that of Rh(111). A higher magnification STM image of the graphene surface is shown in Fig. 7.16b with the unit cell of the moiré superstructure marked by a rhombus. Four distinctive regions corresponding to different apparent height levels can be 


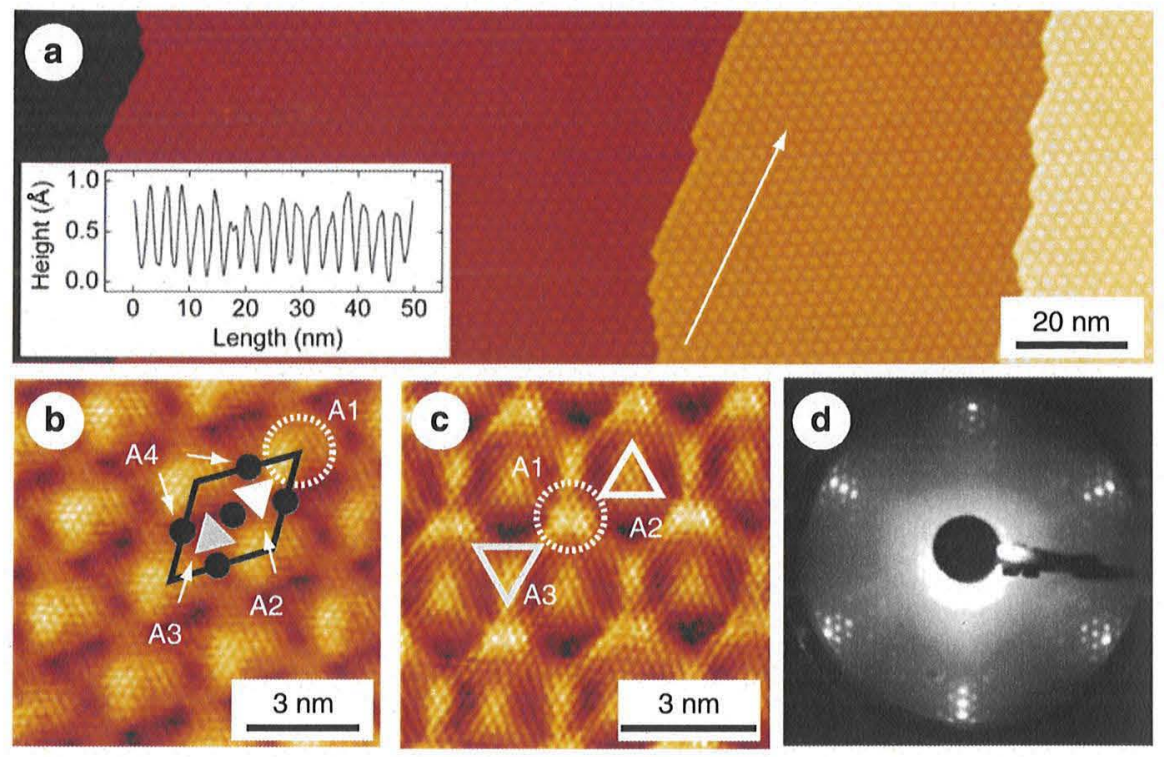

Fig. 7.16 (a) Large-scale STM image of an epitaxial graphene layer on the $\mathrm{Rh}(111)$ surface. Inset shows a height profile measured along the white arrow. (b) High magnification image of the unit cell of the moiré structure. The black rhombus outlines the supercell of the moiré structure. Main surface features are marked in the image: moiré maximum (A1) by a circle, the brighter half-cell (A2) by a white solid triangle, the darker half-cell (A3) by a gray solid triangle, and bridging black minima (A4) by black solid circles. (c) Atomically resolved STM image of the moiré structure of the graphene layer on $\mathrm{Rh}(111)$ taken with different tunneling parameters compared with (b). (d) LEED image of the graphene layer on $\mathrm{Rh}(111)$ taken at a primary electron energy of $58 \mathrm{eV}$. Tunneling parameters for STM images: (a) $U_{\mathrm{T}}=1.12 \mathrm{~V} ; I_{\mathrm{T}}=0.18 \mathrm{nA} ;\left(\right.$ b) $U_{\mathrm{T}}=0.01 \mathrm{~V}$; $I_{\mathrm{T}}=2.56 \mathrm{nA} ;(\mathbf{c}) U_{\mathrm{T}}=0.02 \mathrm{~V} ; I_{\mathrm{T}}=30 \mathrm{nA}$. Data are taken from [117]

distinguished within the moiré supercell: three maxima of different heights (A1, A2, A3) and black minima (A4). The most prominent maxima (A1) are surrounded by six black minima as well as by three less intense maxima A2 and A3. Each of the less intense maxima (A2, A3) is surrounded by three black minima (A4). The different contrast features within the moiré unit cell can be distinguished much better in the high-resolution STM image in Fig. 7.16c recorded at different tunneling conditions compared to Fig. 7.16b. In most regions of the moiré supercell, the graphene sublattice symmetry is broken and only one of the two carbon sublattices is imaged. We attribute this effect to the strong, covalent interaction between the graphene layer and the $\mathrm{Rh}(111)$ substrate.

A more detailed description of the atomic configurations can be carried out on the basis of a simple ball model presented in Fig. 7.3b. Three highly symmetric positions of carbon atoms (or centers of carbon rings) with respect to the underlying Rh lattice can be assigned: $h c p-f c c$ (or atop) sites at the corners of the moiré unit cell, top $f c c$ (or $h c p$ ) and top-hcp (or $f c c$ ) sites in the center of the right and left half-cell of the moiré unit cell, respectively. In the STM image [Fig. 7.16c] 
$h c p-f c c$ regions appear as the bright maxima of the moiré structure (A1), top$h c p$ and top-fcc appear as the two less intense maxima (A2 and A3), exhibiting two different intensity levels (A2 is brighter than A3). In contrast to graphene on $\operatorname{Ru}(0001)[42,43,120]$, in this present study we observe that bright features (local maxima) are surrounded by dark depressions (A4) present in both halves of the moiré unit cell corresponding to the bridge-top areas, which we believe are strongly bonded to the metallic substrate. The full graphene honeycomb lattice is only visible in areas directly on top or adjacent to the A1 maxima (moiré maxima), which we attribute to the weak coupling of these regions of the graphene sheet to the $\mathrm{Rh}$ lattice. The atomic structure of other regions is dominated by the strong covalent interaction with the metal substrate, especially within the black minima (A4), where $\mathrm{C}$ atoms are placed in bridge-top positions. Recent theoretical investigations showed that for graphene on $\mathrm{Ni}(111)$ [80] as well as for graphene on $\mathrm{Ru}(0001)$ [121] such bridge positions are energetically favorable, which might lead to a relatively strong $\mathrm{C}-\mathrm{Rh}$ bonding in these regions. Especially the bridge-top position, which we believe gives rise to the observed black depressions in the STM images, was found to be one of the most energetically stable configurations [80]. Interestingly, the observed pronounced bonding of the bridge-top regions is almost completely suppressed in graphene on $\operatorname{Ru}(0001)[42,43,120]$, where no local height variation within the halfcells of the moiré structure was observed. We also would like to point out that the atomic structure of graphene on $\mathrm{Rh}(111)$ also differs from that of the $h$-BN "nanomesh" on $\operatorname{Rh}(111)[131,132]$.

\subsubsection{Electronic Structure of Graphene on Lattice-Mismatched Surfaces}

In a straightforward way, the degree of interfacial hybridization between graphene $\pi$ and TM $d$ states can be probed in the $\mathrm{C} 1 s \mathrm{X}$-ray absorption experiment. Figure 7.17a shows $\mathrm{C} 1 s$ NEXAFS spectra for graphene layer on $\operatorname{Pt}(111), \operatorname{Ir}(111)$, $\mathrm{Rh}(111)$, and $\mathrm{Ru}(0001)$ together with the corresponding LEED patterns [41]. The spectra are arranged in such a way that from top to bottom the substrate-induced changes to the original graphite spectrum increase, thus providing a direct evidence for the growing chemical interaction between graphene and metals in the series Pt-Ir-Rh-Ru. Evidently, the $4 d$ and $5 d$ metals fall into two different groups as regards bonding to graphene. In the case of $5 d$ metal substrates, the $\mathrm{C} 1 s$ NEXAFS spectrum is weakly affected preserving shapes and energies of the distinctive resonant features A, B, and C. On Pt(111), the influence of the substrate is restricted mainly to the appearance of the step-like feature $\mathrm{A}^{\prime}$ at $284 \mathrm{eV}$ due to the lowering of the Fermi level as a result of the charge transfer at the interface. Very weak chemical bonding of graphene to $\operatorname{Pt}(111)$ cannot force the overlayer uniquely in registry with the substrate, as can be judged from the LEED pattern with segmented rings reflecting different domain orientations of graphene [Fig. 7.17a, lower 
a
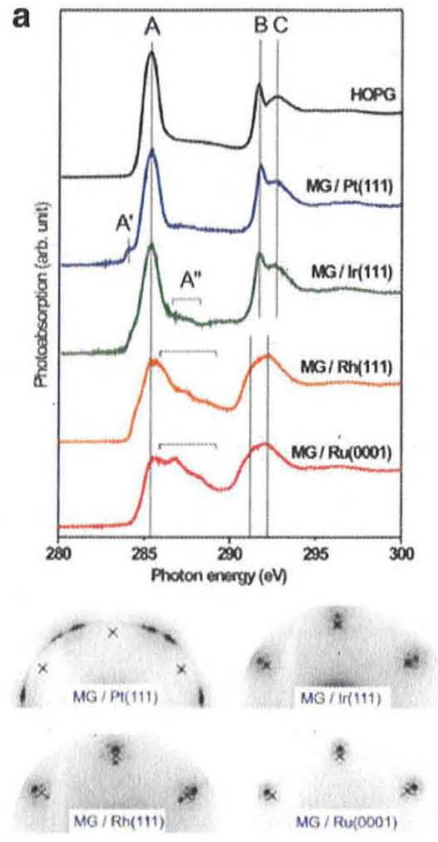

b

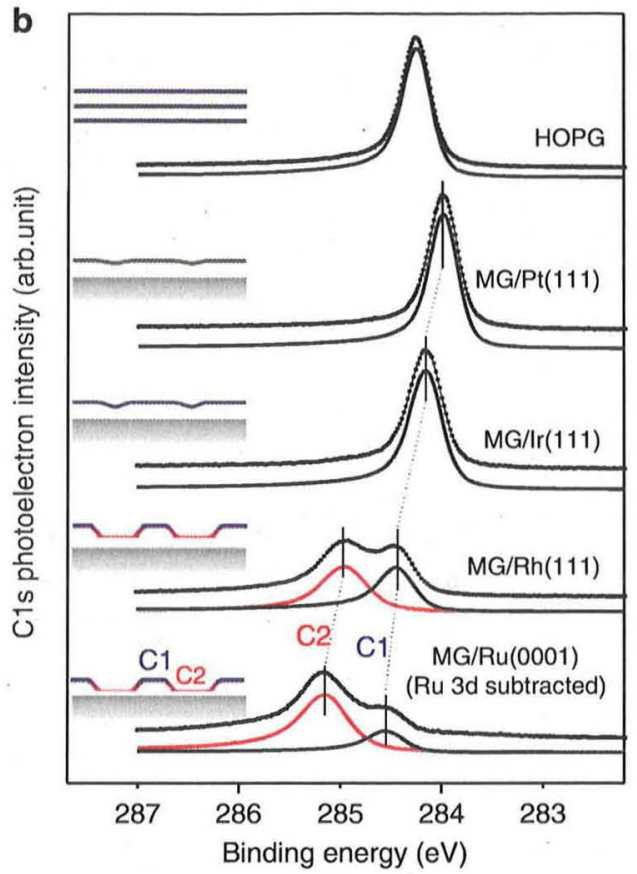

Fig. 7.17 (a) C $1 s$ NEXAFS spectra from graphene layer adsorbed on several lattice-mismatched TM surfaces. The C $1 s$ spectrum from HOPG is shown for comparison. Lower panel shows the corresponding LEED patterns; substrate-related spots are marked with crosses. (b) C $1 \mathrm{~s}$ photoelectron spectra taken in normal emission from graphene layer adsorbed on several latticemismatched TM surfaces. Photon energy is $400 \mathrm{eV}$. For graphene/Ru(0001), the Ru $3 d$ signal is subtracted. Data are taken from [41]

panel] $[41,104,105,122,133,134]$. The detailed structure of possible graphene/Pt moiré patterns has been revealed in a recent combined LEED/LEEM study [107]. On $\operatorname{Ir}(111)$, shoulder $\mathrm{A}^{\prime}$ is already disturbed by weak chemisorption and a new bonding-related $\pi^{*}$ feature $\mathrm{A}^{\prime \prime}$ starts to develop between $286.5 \mathrm{eV}$ and $288 \mathrm{eV}$. A reduction in the $5 d$ occupancy in going from $\operatorname{Pt}\left(d^{9}\right)$ to $\operatorname{Ir}\left(d^{7}\right)$ is probably the main reason for the growing interaction with graphene, since it causes a depopulation of the antibonding states. A slight increase in the bonding strength (as compared to graphene/Pt) results in the occurrence of one principal domain orientation, as reflected in the mismatch-induced moiré pattern in LEED [Fig. 7.17a, lower panel].

In the case of $4 d$ metal substrates ( $\mathrm{Rh}$ and $\mathrm{Ru}$ ), the electronic structure of graphene is strongly disturbed, as reflected in the C $1 s$ NEXAFS spectra [Fig. 7.17a]. Indeed, the spectra of graphene on $\mathrm{Rh}(111)$ and $\mathrm{Ru}(0001)$ resemble the $\mathrm{C} 1 s$ spectrum from graphene on $\mathrm{Ni}(111)$, which is discussed above (Fig. 7.8). Both $\pi^{*}$ and $\sigma^{*}$ resonances are strongly reshaped, and the separation between the $\pi^{*}$ and $\sigma^{*}$ manifolds is visibly reduced. Again, these changes reflect strong TM $4 d$ - graphene $\pi$ orbital mixing and softening of the $\mathrm{C}-\mathrm{C}$ bonds caused by the 
formation of covalent bonds between $\mathrm{C}$ atoms and the substrates. The general trends in the strength of interaction between graphene and late TMs are the following: (1) along the row it increases with decreasing occupancy of the $d$ shell and (2) for the same occupation numbers, it is similarly strong for the $3 d$ and $4 d$ shells, but considerably weaker for the $5 d$ shell. The former trend is due to the gradual depopulation of the antibonding states with reducing $d$ shell occupation. The latter effect can be understood in terms of orbital overlap. In going from $4 d$ to $5 d$ elements, relativistic effects become significant, resulting in contracting the $6 \mathrm{~s}$ orbitals and expanding the $5 d$ orbitals. Probably, the overlap of the graphene $\pi$ states with more diffuse $5 d$ orbitals is worse than with more localized $4 d$ orbitals.

The co-existence of lowered and elevated graphene areas on lattice-mismatched TM substrates (see previous subsection for the exact surface geometry) can be directly observed in a core-level PE experiment. Figure $7.17 \mathrm{~b}$ shows $\mathrm{C} 1 \mathrm{~s} \mathrm{PE}$ spectra from graphene on $\operatorname{Pt}(111), \operatorname{Ir}(111), \operatorname{Rh}(111)$, and $\mathrm{Ru}(0001)$ compared with the reference spectrum from HOPG. The double-peak structure observed for graphene/Rh and graphene/Ru is due to the strong corrugation of graphene: $\mathrm{C} 1$ results from the nonbonding elevated sites, while $\mathrm{C} 2$ represents bonding lowlying areas. The peak-fit analysis reveals that $\mathrm{C} 2$ is considerably broader than $\mathrm{C} 1$ reflecting the effect of gradually varying chemical surrounding of the $\mathrm{C}$ atoms across the bonding sites [41]. There is an obvious correlation between the strength of interfacial chemical bonds probed by NEXAFS [Fig. 7.17a] and the size of the area occupied by the bonding sites [Fig. 7.17b]. On the $5 d$ metal substrates, the bonding with graphene is weak, and the $\mathrm{C} 2$ component is not detected in the C $1 s$ PE spectra, thus implying no pronounced "pores" and weak corrugation. With increasing covalent interaction at the graphene/TM interfaces, component $\mathrm{C} 2$ grows in the spectrum at the cost of $\mathrm{C} 1$, and becomes dominating for the most reactive graphene/Ru interface. Even quantitative information about the effective "pore" sizes for different graphene/TM interfaces can be extracted from the core-level PE spectra if the effects of photoelectron diffraction on the intensity ratio $\mathrm{C} 1 / \mathrm{C} 2$ are properly filtered out [41].

In order to gain a detailed insight into the origin of the bonding between graphene and lattice-mismatched noble metal substrates, photoemission studies have been performed, which provide direct information about the electronic structure of the occupied valence band states. An ARPES photoemission intensity map of the graphene/ $\operatorname{Ir}(111)$ system measured along the $K-\Gamma-K$ direction is shown in Fig. 7.18 [135]. In the ARPES spectra of graphene on $\operatorname{Ir}(111)$, the $\pi$ band with linear dispersion up to $E_{F}$ and the Dirac cone are clearly visible at the $K$ point $[112,135]$. The position of the Dirac point was estimated to be $0.10 \pm 0.02 \mathrm{eV}$ above $E_{F}$, indicating only a slight $p$-doping $[112,135]$. No sign of hybridization of graphene with the electronic bands of $\operatorname{Ir}(111)$ was observed, which is in line with the recent DFT calculations suggesting only a weak bonding [136]. Additionally, Dirac cone replicas and minigaps in the band structure were observed being the consequence of a superperiodic potential imposed by the moiré structure [112,135].

In the case of strongly interacting graphene/ $\mathrm{Ru}(0001)$ system, pronounced changes in the electronic structures occur compared with quasi-free-standing 

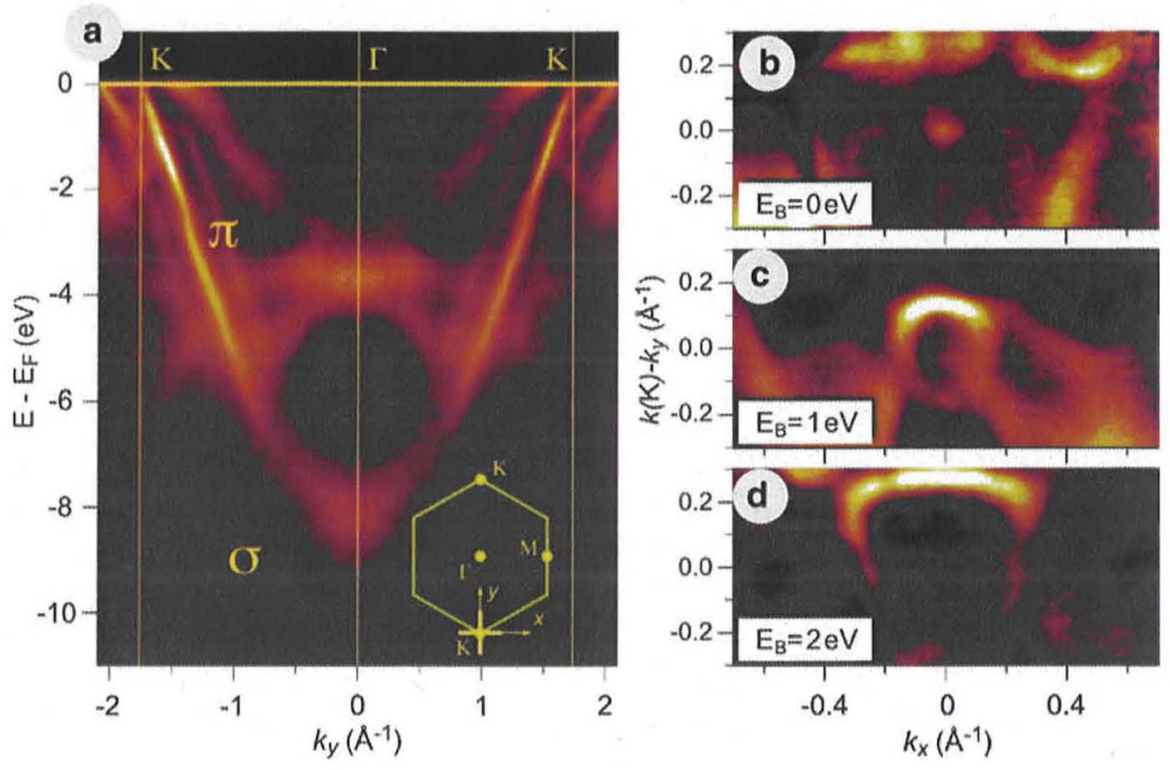

Fig. 7.18 (a) Photoemission intensity map along the $K-\Gamma-K$ direction and (b-d) the constant energy cuts obtained for the graphene/Ir(111) system at $h v=120 \mathrm{eV}$ of photon energy. Data are taken from [135]

graphene or graphene/ $\operatorname{Ir}(111)$. Figure 7.19 shows the measured band structure of graphene $/ \mathrm{Ru}(0001)$ along the $\Gamma-K$ direction of the surface Brillouin zone [124]. At the $K$ point, the $\pi$ band reaches a binding energy of $4.6 \pm 0.1 \mathrm{eV}$, which confirms a strong interaction of graphene with the substrate. The experiment is in good agreement with calculations for a $1 \times 1$ graphene sheet $2.2 \AA$ above the topmost Ru layer with the top-hcp atomic configuration. Similar results were obtained in recent photoemission studies on the strongly bound graphene on $\mathrm{Rh}$ (111) [117]. The difference in binding energy of the $\pi$ states at the $\Gamma$ point in graphene/ $\mathrm{Rh}(111)$ and pure graphite amounts to about $2.3 \mathrm{eV}$, which is close to the value for graphene/Ni(111) and also for graphene $/ \mathrm{Ru}(0001)$. This shift reflects the effect of hybridization of the graphene $\pi$ bands with the Rh $4 d$ bands and, to a lesser extent, with the Rh $5 s$ and $5 p$ states. These results indicate that the bonding strength of the graphene layer on top of the $\mathrm{Rh}(111)$ surface is comparable to that observed in graphene/Ru(0001). However, a considerable local redistribution of the orbital hybridization in graphene/Rh(111) compared to $\mathrm{Ru}(0001)$ or $\operatorname{Ir}(111)$ can be deduced from strong contrast variations observed by STM. 

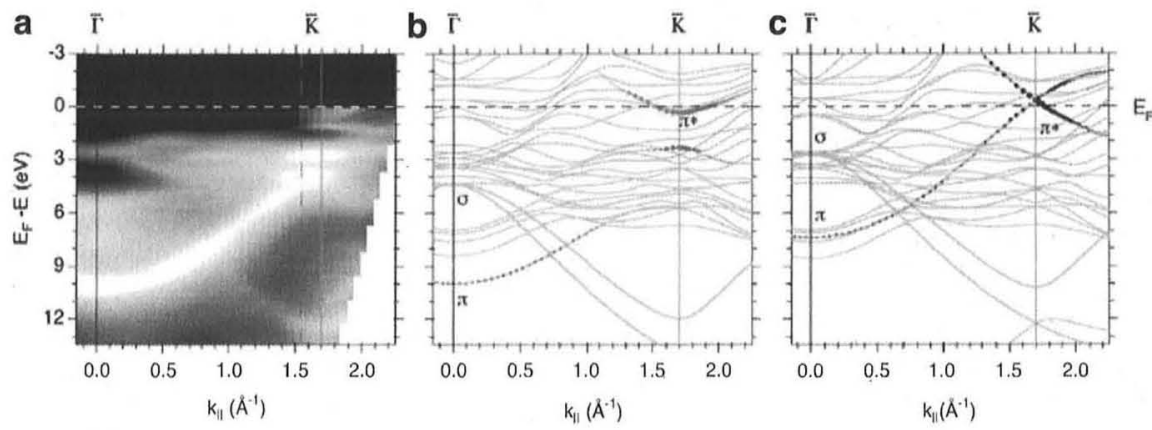

Fig. 7.19 (a) Band structure of the graphene/Ru(001) system measured along the $\bar{\Gamma}-\bar{K}$ direction of the hexagonal Brillouin zone. (b) and (c) DFT of graphene/Ru(0001) for the low $\left[\left(\mathrm{C}_{A}, \mathrm{C}_{B}\right) \sim(\right.$ top,$\left.h c p)\right]$ and high $\left[\left(\mathrm{C}_{A}, \mathrm{C}_{B}\right) \sim(h c p, f c c)\right]$ regions, respectively. The vertical lines at $\bar{K}$ indicate the boundaries of the $1 \times 1$ surface Brillouin zones for Ru (red dashed) and graphene (green solid). The size of the filled circles in (b) and (c) represents the $p_{z}$ weight of the adsorbate atoms on the bands, where blue describes $\mathrm{C}_{A}($ top $)$ and red describes hollow site atoms $\left(\mathrm{C}_{B}\right)$. Black circles depict the average of the two inequivalent adsorbate atoms. Thick yellow curves are guides for the eyes. Data are taken from [124]

\subsection{Hybrid Structures on the Basis of Graphene Layers on Metal Surfaces}

As was shown in previous sections, high-quality graphene layers can be grown on closed packed TM surfaces by means of CVD of hydrocarbons on the surface or segregation of carbon from the bulk. In contrast to the graphite intercalation compounds where the interaction between the graphene layers and the intercalated atoms is dominated by charge transfer, bonding of the graphene layers to the transition metal substrate has a more covalent character due to hybridization of $d$-orbitals with the $\pi$ states of graphene layer. In spite of stronger interactions, alkali or noble metals can be intercalated between graphene layer and the metal substrate. The experimental procedure requires the deposition of intercalation material on the graphene-covered surface followed by the subsequent annealing of the system, which results effective intercalation underneath graphene. While in the case of alkali metals, a strong charge transfer into the antibonding $\pi^{*}$ states is expected [73], particularly intercalation of noble metals leads to a weakening of the graphenesubstrate bonding and the formation of almost isolated graphene layers on the substrate [22, 23, 26, 137-139].

Intercalation of magnetic transition metal atoms such as Co between the graphene overlayer and an e.g. nonmagnetic substrate have still not been reported. Only recent experiments show that at least $\mathrm{Fe}$ may be intercalated between a graphene layer and an $\mathrm{Ni}(111)$ substrate [24]. In an alternative approach, thin magnetic films may be grown on a nonmagnetic substrate and be covered subsequently by graphene. A possible problem here may be chemical reactions of carbon with the transition 
metal substrate induced by the high temperatures needed for the cracking of the propene molecules. For Ni surfaces, respective interactions have not been observed. The situation, however, may change for $\mathrm{Co}$ and $\mathrm{Fe}$, where the tendency of carbide formation is much larger than for $\mathrm{Ni}$.

Another interesting scenario is the growth of thin transition metal films on top of a graphene monolayer. If ordered overstructures can be grown without intercalation or destruction of the graphene layer, then even multilayer structures of graphene and transition metals may be synthesized. In fact, carbide formation may be suppressed if the temperature is kept low enough. Such hybrid structures (FM/graphene/FM) were predicted to be a perfect spin filter due to the perfect in-plane lattice constants match and that their electronic structures overlap in reciprocal space for one spin direction only. On the contrary, transition-metal adsorbate may tend to island growth on graphene at low temperatures. In fact, island growth has been reported for $\mathrm{Mn}$, $\mathrm{Cr}$, and $\mathrm{V}$ [140], while the formation of a monolayer was achieved for $\mathrm{Ru}$ on $\mathrm{C}(0001)$ [141].

\subsubsection{Intercalation-like Systems}

The first attempt to prepare a graphene-based intercalation-like system was reported in the work by Nagashima et al. [73], where intercalation of alkali atoms (Cs, $\mathrm{K}$, Na) underneath graphene layer on $\mathrm{Ni}(111)$ was performed. Efficient intercalation of alkali-metals underneath of the graphene layer was observed already at room temperature. Alkali-metal intercalation is accompanied by a modification of dispersion of the valence band states of graphene due to the increasing of the distance between graphene layer and $\mathrm{Ni}$, and consequently, the weakening of the hybridization strength between graphene $\pi$ and $\mathrm{Ni} 3 d$ valence band states. This effect is reflected in the valence band photoemission spectra of this system by a strong modification of dispersion of the graphene $\pi$ states around the $K$ point of the Brillouin zone (Fig. 7.20). The electronic structure of the obtained graphenebased intercalation-like system is very similar to the one of the pristine graphite crystal. It was found that intercalation of alkali metals underneath graphene layer is energetically favorable, leading to the weakening of the bonding between graphene and substrate. The main results of these experiments were recently confirmed and refined in work [74]. Similar effects were observed upon intercalation of the divalent rare-earth metals, such as Eu and $\mathrm{Yb}$ [142].

Later on, the main interest in the graphene-based intercalation-like systems shifted to the investigation of the possibility to recover the original free-standing graphene-like electronic structure, where the Dirac cone is fully or partially restored. From the electronegativity point of view, it is possible via intercalation of noble metals, such as $\mathrm{Cu}, \mathrm{Ag}$, or $\mathrm{Au}$. The earlier studies of $\mathrm{Cu}$ - and $\mathrm{Au}$-intercalation underneath graphene on $\mathrm{Ni}(111)$ performed by means of ARPES and high-resolution electron-energy-loss spectroscopy (HREELS) demonstrate that it is possible to fully decouple electronic structures of graphene and substrate [22, 23, 137-139] 

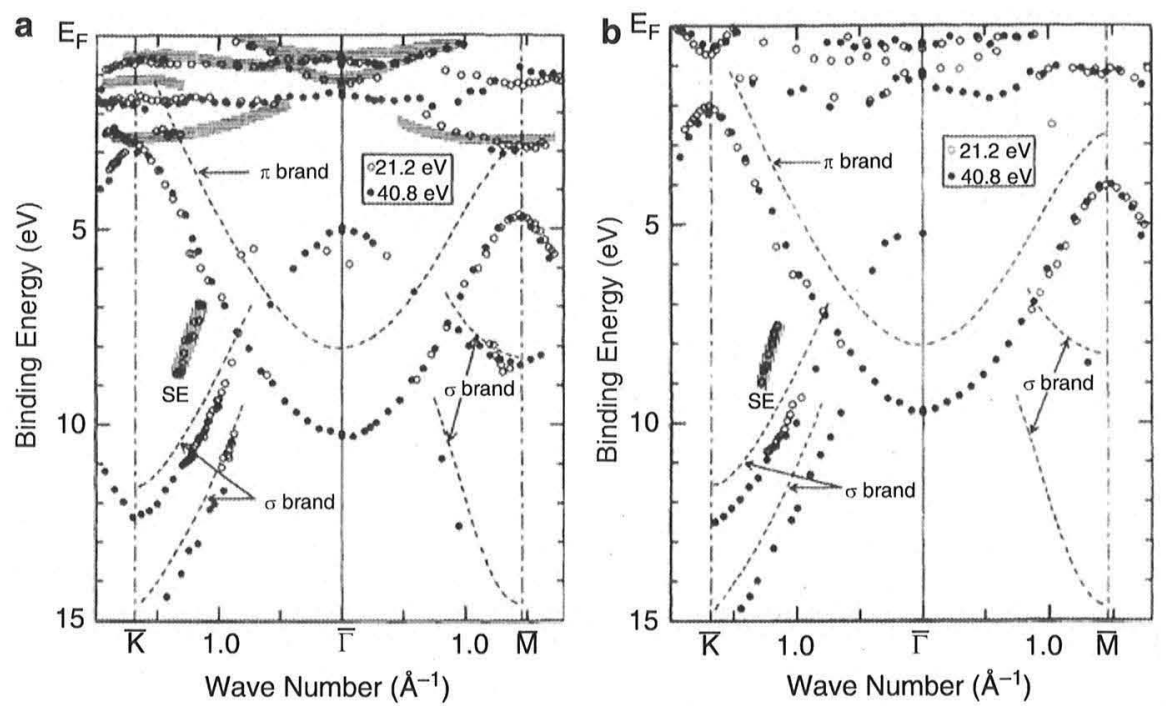

Fig. 7.20 Experimental band dispersion of (a) graphene/ $\mathrm{Ni}(111)$ and (b) the graphene/ $\mathrm{Na} / \mathrm{Ni}(111)$ system. Shaded bands in (a) near $E_{\mathrm{F}}$ indicate the dispersion relations of the Ni $3 d$ bands for the clean $\mathrm{Ni}(111)$ surface. Experimental dispersions of the bulk graphite are also shown by broken lines. Data are taken from [73]

(Fig. 7.21). Valence-band PES spectra taken in normal-emission geometry at $50 \mathrm{eV}$ photon energy are shown in Fig. 7.21a and demonstrate the intercalation of thin layers of $\mathrm{Cu}(4 \AA$ and $12 \AA)$ underneath graphene layer on $\mathrm{Ni}(111)$ [23]. In the energy distribution curves, features originating from the $\pi$ states of graphene and the $3 d$ states of $\mathrm{Ni}$ can be readily distinguished. Upon deposition of copper on graphene/Ni(111), the PE intensity of the $\pi$ graphene-derived states decreases, but remains clearly visible. Annealing of the $\mathrm{Cu} / \mathrm{graphene} / \mathrm{Ni}(111)$ system leads to an increase of intensity of the $\pi$ states of graphene at the expense of the $\mathrm{Cu} 3 d$ signal and a shift of the $\pi$ graphene-derived band toward lower binding energies. Thereby, the intensity of the Ni $3 d$ emission is almost unaffected by the annealing. The same behavior was observed for the thicker copper pre-deposited layers. Such changes of the intensities of the valence-band features of the studied system were assigned to the intercalation of thin $\mathrm{Cu}$ layers in the space between graphene and $\mathrm{Ni}$ substrate. The energy shift of the $\pi$ band is caused by a change of chemical bonding between the graphene layer and the substrate through intercalation of $\mathrm{Cu}$ [23]. These observations were confirmed by STM and LEED, where a slight misorientation of the weakly bonded regions of graphene was detected [23].

Energy dispersions of the graphene-derived bands in the Brillouin zone of graphene are plotted in Fig. 7.21b for the graphene/Cu/Ni(111) system (different thicknesses of copper layer are marked in the figure) [23]. It was found that for this intercalation-like graphene-based system the measured energy bands are shifted toward lower binding energies as compared to their location for the 
a

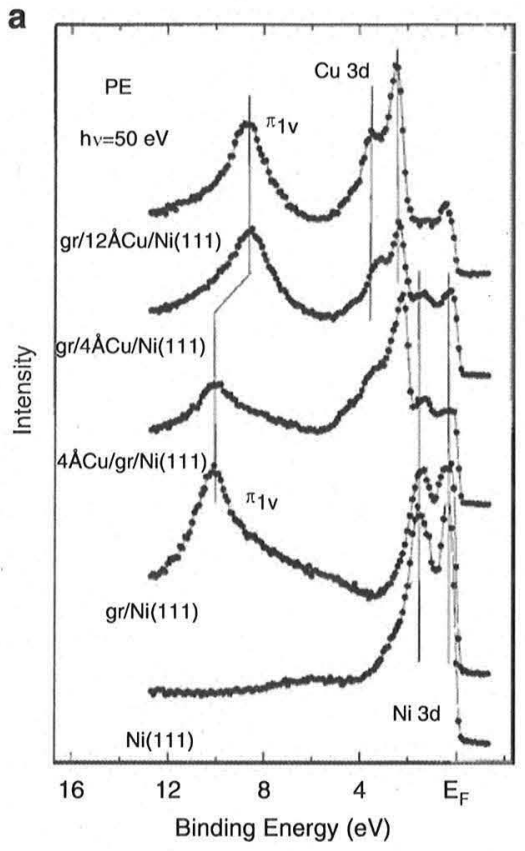

b

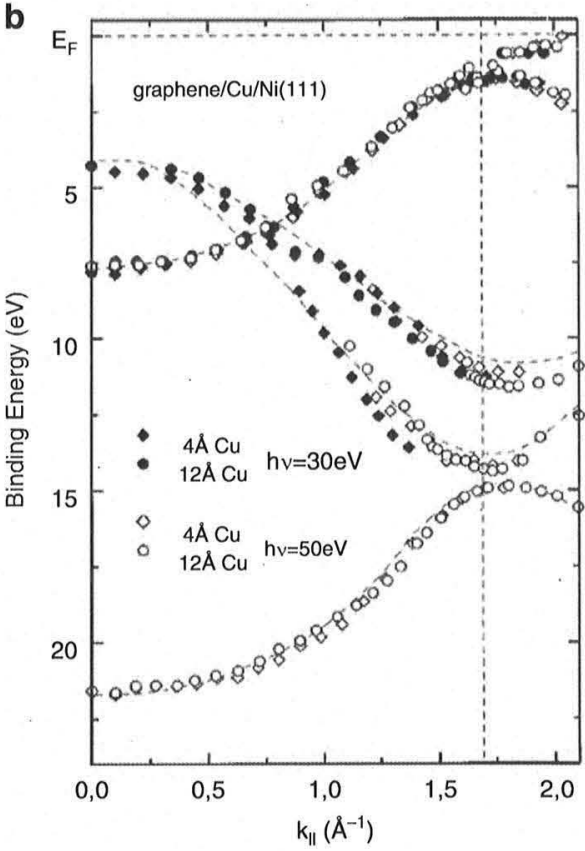

Fig. 7.21 (a) Normal emission PE spectra demonstrating the process of $3 d$ metal intercalation underneath graphene layer on $\mathrm{Ni}(111)$ : of the clean $\mathrm{Ni}(111)$ surface, a graphene/Ni(111), $4 \AA \mathrm{Cu} / \mathrm{graphene} / \mathrm{Ni}(111)$, as well as the graphene/4 $\AA \mathrm{Cu} / \mathrm{Ni}(111)$ and the graphene/12 $\AA \mathrm{Cu} /$ $\mathrm{Ni}(111)$ systems. (b) Experimental band structure of the graphene/Cu/Ni(111) system. For comparison, the band structure of pristine graphite is shown by dashed lines. Data are taken from [23]

graphene/Ni(111) system; they approach the energies known for pristine graphite. Only for the graphene/Cu/Ni(111) system a splitting of the $\pi$ states is observed around the $K$ point: One spectral feature shifts toward higher binding energies for angles corresponding to emission from the second Brillouin zone; a second feature continues to shift up to an energy closest to $E_{\mathrm{F}}$, but differs from that of the graphene/ $\mathrm{Ni}(111)$ system. These features can be well defined in the second Brillouin zone, but cannot be distinguished in the first Brillouin zone. The same behavior of the graphene-derived valence-band states was later observed for the system obtained via Ag-intercalation [138].

An interesting case was considered in $[22,26,139]$, where intercalation of $\mathrm{Au}$ underneath graphene was investigated by means of STM and ARPES. In these works, the amount of intercalated Au was estimated to be $1 \mathrm{ML}$ ( $\mathrm{ML}=$ monolayer) independent on the thickness of the predeposited metal, which is different from the $\mathrm{Cu}$ and $\mathrm{Ag}$ intercalation where different thicknesses of pre-deposited metal were intercalated [23, 137, 138]. In [22], an almost complete restoration of the free-standing graphene band structure along the $\Gamma-M$ direction of the Brillouin 
zone was observed for the graphene/Ni(111) system after Au-intercalation that was also confirmed by the accompanied HREELS studies. These results were recently confirmed by the more systematic studies of the graphene/Au/Ni(111) system [139], where the full restoring of the Dirac-cone at the Fermi level was observed (within the accuracy of $25 \mathrm{meV}$ ).

The systematic studies of the gold intercalation underneath the graphene layer on $\mathrm{Ru}(0001)$ were recently performed by means of ARPES [26]. For this system, the Fermi surface appears to be point-like; however, a close look at the dispersion of the graphene-derived $\pi$ states around the Fermi level [Fig. 7.22a; left-hand side, lower panel] reveals that the Dirac point is $\approx 150 \mathrm{meV}$ above $E_{\mathrm{F}}$, which is consistent with a small $p$-doping of the graphene layer in the graphene/Au/Ru(0001) system. A similar $p$-doping of graphene was recently observed in the case of deposition and annealing of a thin $\mathrm{Au}$ layer on the graphene monolayer on $\mathrm{SiC}(0001)$ [25]. In order to obtain an information about the Dirac crossing point, the electron doping via deposition of $\mathrm{K}$ atoms was performed, and the clear energy gap of about $200 \pm 60 \mathrm{meV}$ was observed, which is independent of the amount of deposited K (Fig. 7.22). It was proposed that the band gap in the graphene/ $\mathrm{Au} / \mathrm{Ru}(0001)$ system results from a symmetry breaking of the two carbon sublattices in the graphene layer, which results in a weak breaking of the chiral symmetry, inducing a weak but finite intensity of the left band along the $\Gamma-K$ direction, between the two arrows in Fig. 7.22c [143]. The ratio of the left to right band intensities in Fig. $7.22 \mathrm{c}$ is about 35 , which agrees with theoretical predictions for the size of a gap of $200 \mathrm{meV}$ [143]. For the $2 \mathrm{ML}$ graphene/Ru(0001), there is no equivalent observable intensity [between the arrows in Fig. 7.22b], consistent with the lack of a gap at the Dirac point.

\subsubsection{Growth of Noble Metal Clusters on Graphene Moirè}

Highly uniform graphene moiré structures can be used as templates for the growth of uniform cluster arrays with nearly perfect long range order. This idea was first experimentally tested for graphene moiré on the $\operatorname{Ir}(111)$ surface allowing the preparation of Ir cluster superlattices [40]. Recently, further experiments with graphene/Ir(111) system were performed aiming to extend the row of superlattice forming materials $[128,144]$. Figure 7.23 displays STM topographs recorded on graphene/ $\mathrm{Ir}(111)$ after deposition $0.2-0.8 \mathrm{ML}$ of various metals at $300 \mathrm{~K}$. Deposited metal clusters are pinned to graphene and the preferred regions of the cluster adsorption at $300 \mathrm{~K}$ were identified as $h c p$ areas of the moiré suprecell where the carbon ring center of graphene is located above $h c p$ sites of the underlying $\operatorname{Ir}(111)$. During initial deposition, the $h c p$ areas are filled by metal clusters with a relatively uniform size until all moiré unit cells are filled. Upon further deposition, metal clusters start to grow in size, but a narrow size distribution is preserved. LDA calculations showed that $s p^{2}$ to $s p^{3}$ rehybridization of carbon atoms in the graphene layer underneath Ir clusters is responsible for the cluster adsorption [136]. Carbon atoms were shown to form strong bonds either to Ir surface atoms of the 
a $\mathrm{K}_{\mathrm{x}}\left(\AA^{-1}\right)$
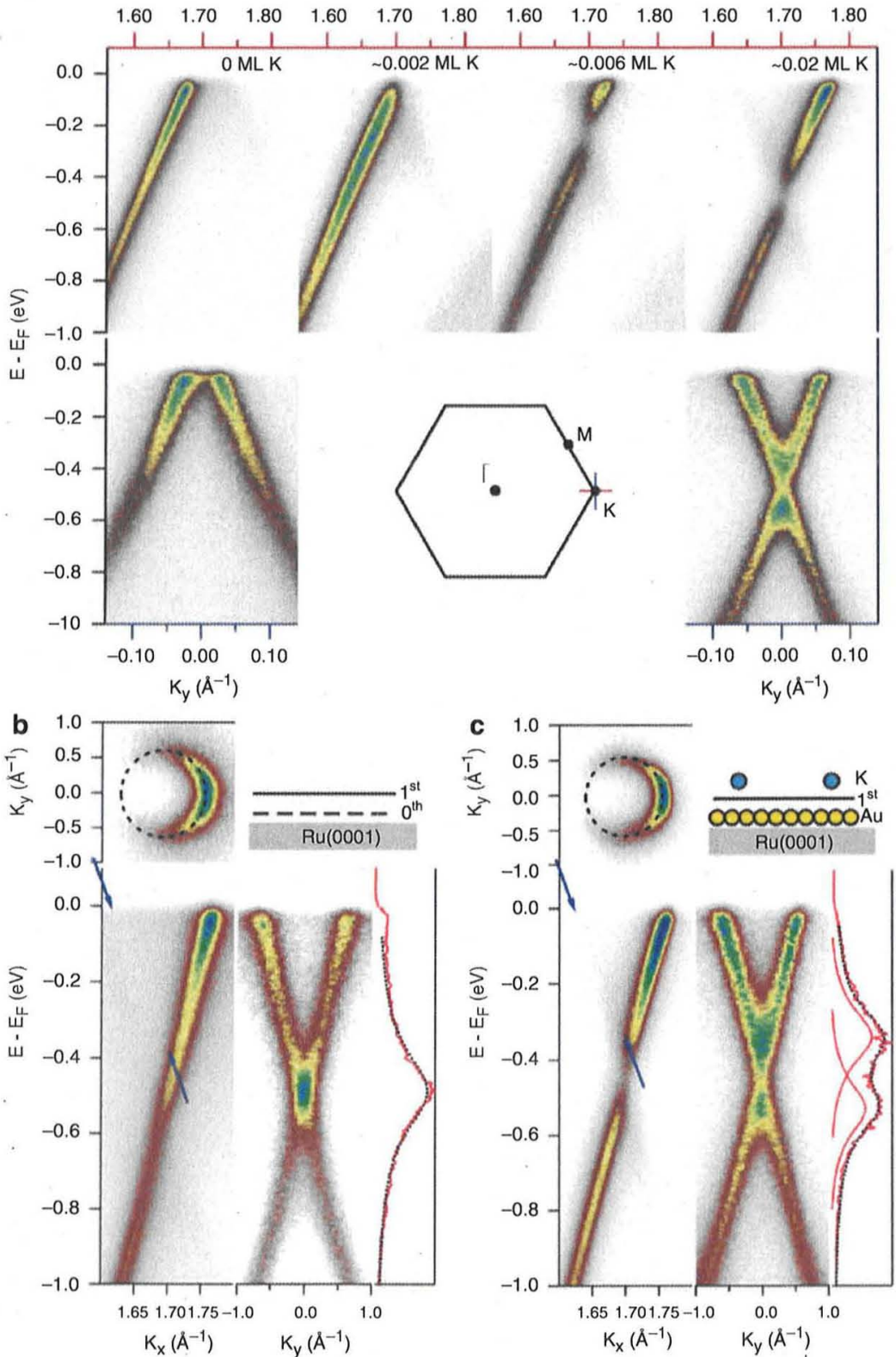

Fig. 7.22 (a) A series of photoemission intensity maps around the $K$ point of the Brillouin zone of the $1 \mathrm{ML}$ graphene/Au/Ru(0001) system for clean (left column) and progressive doping with potassium. The upper and lower rows are taken along the two orthogonal directions in the reciprocal space as indicated by the red and black lines at the $K$ point in the Brillouin zone (inset). Comparison of the spectral function of (b) $2 \mathrm{ML}$ graphene/Ru(0001) and (c) graphene/1 ML $\mathrm{Au} / \mathrm{Ru}(0001)$ is shown in the lower panel together with corresponding PE intensity profiles at the $K$ point. Data are taken from [26] 

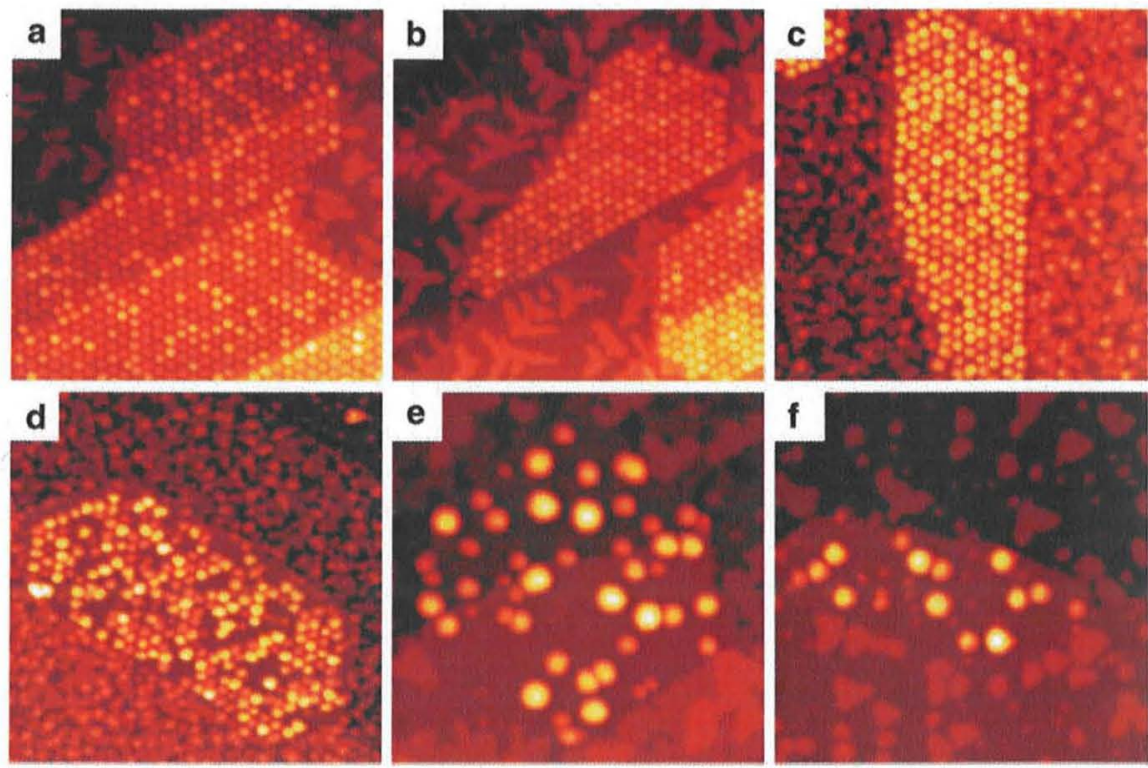

Fig. 7.23 $700 \AA \times 700 \AA$ STM images of the $\operatorname{Ir}(111)$ surface covered by graphene flakes after the deposition of various metals at $300 \mathrm{~K}$. (a) $0.20 \mathrm{ML}$ Ir, average cluster size $S_{\mathrm{av}}=17$ atoms; (b) $0.25 \mathrm{ML} \mathrm{Pt}, S_{\mathrm{av}}=22$ atoms; (c) $0.44 \mathrm{ML} \mathrm{W}, S_{\mathrm{av}}=38$ atoms; (d) $0.53 \mathrm{ML} \mathrm{Re}, S_{\mathrm{av}}=60$ atoms; (e) $0.77 \mathrm{ML} \mathrm{Fe}, S_{\mathrm{av}}=420$ atoms; (f) $0.25 \mathrm{ML} \mathrm{Au}, S_{\mathrm{av}}=100$ atoms. Data are taken from [144].

substrate or to the Ir atoms of the clusters resulting in buckling of the graphene layer. However, as can be seen in the STM images not all metals form a cluster superlattice on graphene/Ir(111). Ir and Pt form superlattices of high perfection [Fig. 7.23a, b], whereas for Fe [Fig. 7.23e] and Au [Fig. 7.23f] no superlattice can be realized at $300 \mathrm{~K}$ [144]. In case of $\mathrm{Re}, \mathrm{Fe}$, and $\mathrm{Au}$, the superlattice formation can be efficiently improved either by lowering the deposition temperature or clusterseeding. In the latter case, Ir seed clusters are first created in nearly all moiré unit cells, which act as nucleation centers during further metal deposition at $300 \mathrm{~K}$ yielding highly uniform metal cluster superlattices with Ir cores [128]. Most of the investigated metal cluster superlattices on graphene//r(111) were found to be stable up to $400 \mathrm{~K}$. More recently, the suitability of the graphene/Ru(0001) moiré for the fabrication of uniform cluster lattices of Pt has been tested [48-50]. Pt clusters were found to preferably occupy locations corresponding to the brighter of the two moiré minima within the moiré unit cell, which are identified as the $f c c$ regions [50]. Upon growth at 140-180 K, Donner et al. [50] prepared highly uniform periodic arrays of monodisperse Pt clusters, which were found to be structurally stable up to room temperature. However, the perfection of the prepared periodic arrays of $\mathrm{Pt}$ clusters on graphene/ $\mathrm{Ru}(0001)$ seems to vary strongly depending on the deposition temperature, Pt deposition rates, as well as deposited amount of Pt [48-50]. Such highly ordered cluster arrays of noble metals are of interest for possible applications in nanocatalysis. 

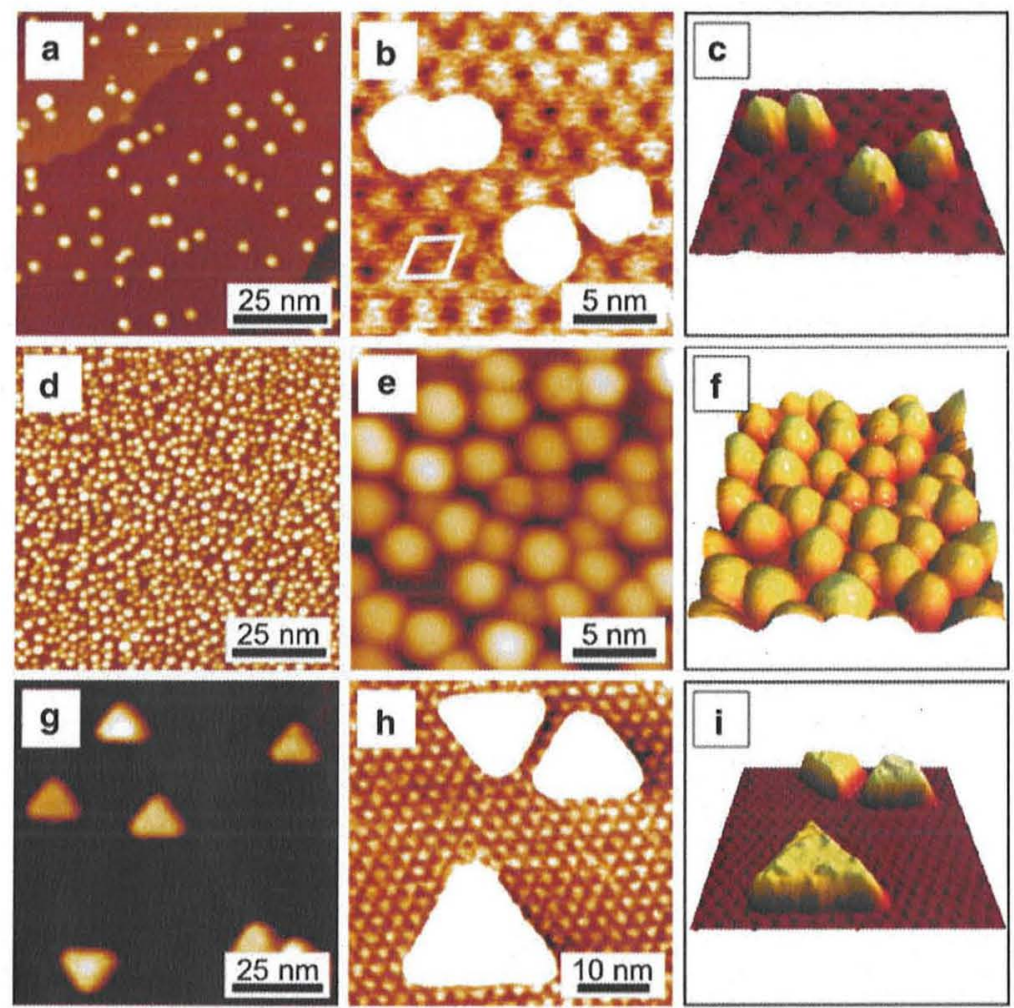

Fig. 7.24 (a-b) Topographic STM images obtained after the deposition of nominally $0.25 \mathrm{ML}$ $\mathrm{Ni}$ at $150 \mathrm{~K}$ on graphene on $\mathrm{Rh}(111)$. The moiré superstructure unit cell in (b) is outlined by a rhombus. (c) Rendered perspective image of the STM topograph presented in (b). (d-e) Topographic STM images obtained after the deposition of nominally $1.50 \mathrm{ML} \mathrm{Ni}$ at $150 \mathrm{~K}$ on graphene on $\mathrm{Rh}(111)$. (f) Rendered perspective image of the STM topograph presented in (e). (gh) STM topograph of nominally $0.90 \mathrm{ML} \mathrm{Ni}$ on graphene/ $\mathrm{Rh}(111)$ deposited at room temperature. (i) Rendered perspective image of the STM topograph presented in (h). Tunneling parameters for STM images: (a-c) $U_{\mathrm{T}}=1.78 \mathrm{~V} ; I_{\mathrm{T}}=0.11 \mathrm{nA} ;(\mathbf{d}) U_{\mathrm{T}}=1.20 \mathrm{~V} ; I_{\mathrm{T}}=0.35 \mathrm{nA} ;(\mathbf{e}-\mathbf{f})$ $\left.U_{\mathrm{T}}=1.28 \mathrm{~V} ; I_{\mathrm{T}}=1.53 \mathrm{nA} ;(\mathrm{g}) U_{\mathrm{T}}=0.90 \mathrm{~V} ; I_{\mathrm{T}}=0.88 \mathrm{nA} ;(\mathbf{h}-\mathbf{i}) U_{\mathrm{T}}=1.54 \mathrm{~V} ; I_{\mathrm{T}}=1.15 \mathrm{nA}\right)$. Data are taken from [51]

\subsubsection{Growth of Magnetic Metal Clusters on Graphene Moirè}

Fabrication of ordered arrays of magnetic clusters is of a particular interest with respect to possible technological applications in magnetic data storage. Graphene moiré on $\mathrm{Rh}(111)$ has recently been used as a template for the growth of $\mathrm{Ni}$ clusters [51]. Figure 7.24a, b shows STM topographs of $0.25 \mathrm{ML}$ of Ni deposited on graphene/Rh(111) at $150 \mathrm{~K}$. Large terraces and steps of graphene/Rh(111) covered with Ni nanoclusters (NCs) can be clearly distinguished. The NCs show mostly hemispherical shapes with at least some cluster edges, which appear to be oriented 
along the in-plane $\langle 1 \overline{1} 0\rangle$ direction. The apparent height of the clusters was measured to be $0.85 \pm 0.1 \mathrm{~nm}$, which roughly corresponds to four $f c c$ (111) planes of Ni. The clusters are loosely bound to the graphene surface at room temperature and could easily be swept away by the STM tip during scanning, which is in row with other reports [50]. The diameter distribution of the clusters is very narrow with the average cluster diameter being $3.1 \mathrm{~nm}$. Most of the $\mathrm{Ni}$ clusters were found to be located on the regular grid showing a quite high unit cell occupation probability for the deposited amount. However, the nucleation of NCs obviously occurs at two different regions within the moiré unit cell (top- $f c c$ and top- $h c p$ ). The randomized occupation can be attributed to the fact that the deposition temperature of $150 \mathrm{~K}$ is below the optimum temperature. Upon increasing Ni coverage, no visible order of the cluster arrangement can be realized. Figure $7.24 \mathrm{~d}-\mathrm{f}$ shows the surface morphology after the deposition of $1.50 \mathrm{ML}$ of $\mathrm{Ni}$ at $150 \mathrm{~K}$. Ni clusters exhibit a distinctly different size distribution compared with that at lower coverages. The lateral size of the clusters is more spread.

$\mathrm{Ni}$ deposited at room temperature exhibits a completely different growth mode compared with deposition at $150 \mathrm{~K}$ [51]. Instead of small compact NCs, Ni forms triangular-shaped islands with their edges roughly aligned with the close packed $<1 \overline{1} 0>$ directions of the $\mathrm{Rh}(111)$ substrate as shown in Fig. $7.24 \mathrm{~g}-\mathrm{i}$. The existence of large islands nucleated at terraces indicates that $\mathrm{Ni}$ atoms are highly mobile on the graphene surface at room temperature. On the other hand, it is remarkable that, in spite of the weak bonding strength between $\mathrm{Ni}$ and graphene surface, the moire structure imposes registration and orientation on the $\mathrm{Ni}$ nanostructures. The average apparent height of the islands is $1.8 \mathrm{~nm}$ and the size defined by the length of the edges is ranging from about $5 \mathrm{~nm}$ to about $18 \mathrm{~nm}$. The surface of the islands is rather flat. The two different orientations can be attributed to the initial nucleation taking place either at a top-hcp and top-fcc site. The long edges of the islands are not always exactly aligned with the high-symmetry $<1 \overline{1} 0>$ directions of $\operatorname{Rh}(111)$. Some of the islands are oriented with an angle of up to $\pm 10^{\circ}$ with respect to these high-symmetry directions. Clockwise-rotated and counterclockwise-rotated islands are found to occur with equal probability. Since the magnetic anisotropy depends on shape and size of the cluster, the deposition temperature is the key parameter to play with in order to control the magnetic properties of the nanoclusters.

\subsubsection{Chemical Functionalization of Graphene on Transition Metal Surfaces}

One of the main routes towards the modification of graphene electronic structure in a controllable way is the chemical functionalization by atoms and molecules, i.e., the process of creating covalent bonds between graphene and adsorbates. The general principles of chemical functionalization of graphene and recent achievements in this field are discussed recently in the review by Boukhvalov and Katsnelson [29]. 
a

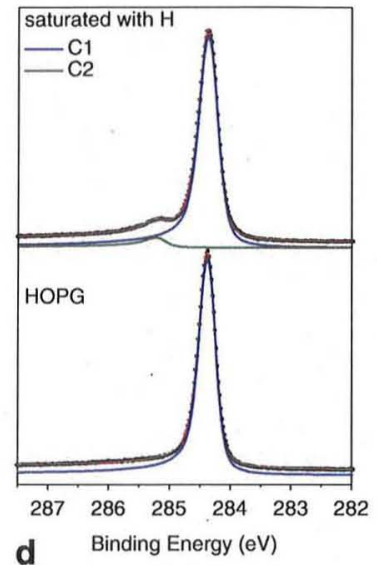

b

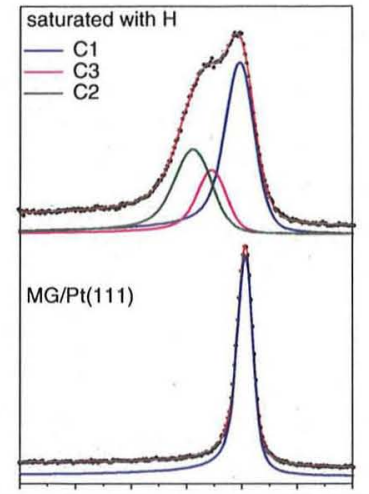

$\begin{array}{lllllll}288 & 287 & 286 & 285 & 284 & 283 & 28\end{array}$ Binding Energy (eV)
C

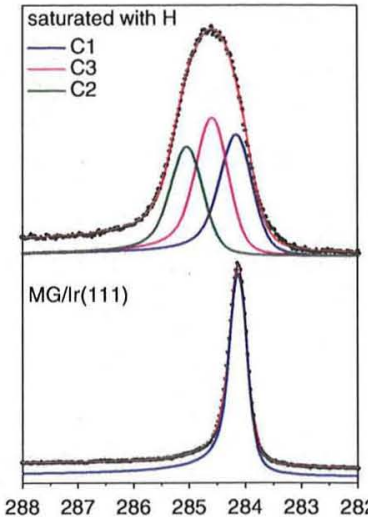

$\begin{array}{lllllll}288 & 287 & 286 & 285 & 284 & 283 & 282\end{array}$ Binding Energy (eV)

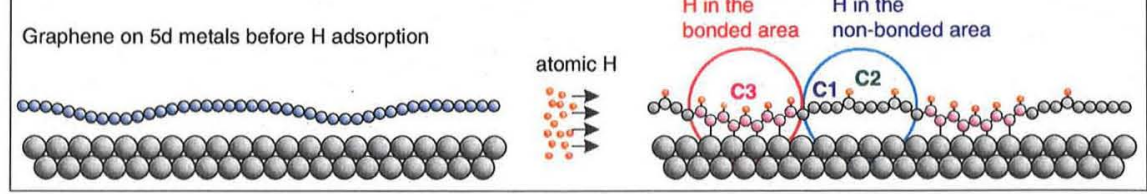

Fig. 7.25 C $1 s$ PE spectra taken with $h v=400 \mathrm{eV}$ from pristine and fully hydrogenated (a) graphite and graphene on (b) $\mathrm{Pt}(111)$ and (c) $\operatorname{Ir}(111)$. (d) Schematic representation of the hydrogenation process for graphene on $5 d$ metal surfaces ( $\mathrm{Ir}, \mathrm{Pt}$ ). Substrate atoms are shown by large circles, $\mathrm{C}$ atoms by middle-size circles, $\mathrm{H}$ atoms by small circles. Data are taken from [145]

In comparison with the free-standing graphene, graphene/metal interfaces may be more advantageous for the formation of covalent bonds with atomic and molecular species, since the interaction with the substrate can weaken the lateral $\mathrm{C}-\mathrm{C}$ bonds and "activate" graphene. This effect can be demonstrated by the example of chemical functionalization of graphene induced by atomic hydrogen. In the graphene/ $\operatorname{Ir}(111)$ system, a gradual $\mathrm{H}$ adsorption has been shown to result in a controllable opening of the band gap due to the $s p^{2}$-to-s $p^{3}$ transition in the more bonding parts of the graphene/Ir(111) moiré [114].

The influence of the chemical nature of the underlying metal on the ability of graphene to adsorb atomic $\mathrm{H}$ has been recently studied for a variety of graphene/metal interfaces [145]. As can be judged from the C $1 s$ PE spectra shown in Fig. 7.25a-c, the same $\mathrm{H}$ treatment of different graphitic substrates results in very different $\mathrm{H}$ uptake values. Here, different spectral components correspond to the $\mathrm{C}$ atoms in the flat $\left(s p^{2}\right)$ coordination $(\mathrm{C} 1)$, directly bonded to $\mathrm{H}(\mathrm{C} 2)$ and directly bonded to the metal substrate in the buckled $\left(s p^{3}\right)$ coordination (C3). Without a reactive substrate, the saturated $\mathrm{H}$ coverage is relatively low, while for graphene on a reactive metal substrate, like $\operatorname{Pt}(111)$ and $\operatorname{Ir}(111)$, the $\mathrm{H}$ uptake value can increase significantly. In Fig. 7.25d, the process of $\mathrm{H}$ adsorption on a nano-patterned graphene layer weakly bound to a $5 d$ substrate is schematically illustrated. The 
more bonding graphene patches (pores) are able to achieve $50 \% \mathrm{H}$ coverage by forming graphene-like structures [146]. This process becomes possible due to the strengthening of the $\mathrm{C} 2 p_{z}-\mathrm{TM} 5 d$ bonds, which can involve the unpaired electrons released upon breaking the $\mathrm{C}-\mathrm{C}$ bonds in the course of $\mathrm{H}$ adsorption. The total area of more strongly bonding graphene sites (pores) is higher for graphene on Ir than on $\mathrm{Pt}$, thus resulting in the higher $\mathrm{H}$ coverage. The nonbonding graphene areas are much more passive, and hydrogenation of these sites is expected to be similar to that of HOPG. For the already strongly bound and lattice matched interfaces (such as graphene/Ni) the mechanism of $\mathrm{H}$ adsorption is different, since further strengthening of the $\mathrm{C}-\mathrm{Ni}$ bonds is impossible. In this case, the unpaired electrons released upon destroying the $\mathrm{C}-\mathrm{C}$ double bonds by $\mathrm{H}$ adsorption are supposed to form dangling bonds on graphene, thus hampering further $\mathrm{H}$ adsorption [145].

\subsection{Conclusions and Outlook}

With all the recent and well-justified excitement about the unusual properties of graphene, it is often forgotten that graphene films on metal surfaces were studied more than forty years ago. Sometimes, they were considered an unavoidable nuisance, for example in the process of cleaning nickel and other transition metal surfaces in ultrahigh vacuum experiments, or an outright problem, in the context of catalyst poisoning through a carbon layer. In the course of such studies, the geometric and electronic structure of these "single layer graphite" films was carefully studied by means of modern surface science techniques, and even the distinction between strongly bound layers and an almost free-standing film, created by intercalation of noble metals between graphene and the transition metal substrate was examined. While the discovery of the unusual electronic and transport properties in graphene in $2005[147,148]$ centered on graphene flakes on insulating $\left(\mathrm{SiO}_{2}\right)$ or semiconducting $(\mathrm{SiC})$ surfaces, renewed attention has returned to graphene on metals. Many interesting discoveries have been made, as documented in this chapter. It was shown that graphene films can be grown with unprecedented structural perfection, over micrometers and larger, on substrates such as iridium and rhodium. Bonding of graphene to lattice-matched ( $\mathrm{Ni}, \mathrm{Co})$ and mismatched $(\mathrm{Ru}, \mathrm{Rh}, \mathrm{Pt}, \mathrm{Ir})$ metals has been analyzed, and the emergence of moiré patterns with large differences in bonding strength in the supercell was observed. The reasons for the large differences in bonding strength of graphene to $\mathrm{Ni}$ and $\mathrm{Co}$, on the one hand, and the $5 d$ metals, on the other, are still a matter of discussion. As far as fundamental studies of electronic effects are concerned, it is true that the preparation and examination of graphene on metals suffers from a serious disadvantage, in that transport experiments, which are at the center of attention (e.g. the anomalous quantum Hall effect) are not feasible. However, this disadvantage is partly compensated by the recent discovery that graphene growth on metals may be the only viable pathway toward the mass production of large-scale monolayer films for deposition on various substrates, for example as transparent electrodes. 
Clearly, much more work is necessary (and in all likelihood, already under way, given the enormous economic stakes involved) to optimize graphene growth and the techniques to lift if off a suitable metal substrate.

The study of graphene on metal substrates has much more to offer, however. The fact that the moiré structure formed by graphene on $\operatorname{Ir}(111)$ gives rise to a template for the growth of clusters with a narrow size dispersion, and with perfect ordering across the surface has come as a complete surprise. Such self-organized cluster growth systems lend themselves almost naturally to investigations of size-dependent electronic structure, general physical properties, and adsorption or catalytic behavior; moreover, in $3 d$ metal clusters, their magnetic properties promise to be an exciting field of study. Here at least, growth on a metal surface does not inhibit investigations as long as the latter is not ferromagnetic, e.g. in the case of iridium.

This brings us to consider the investigation of graphene-ferromagnet interfaces: theoretical studies predict a few-layer film of graphene on either $\mathrm{Ni}$ or Co to act as a highly efficient spin filter, because of the difference in overlap between the minority and majority spin states and the graphene bands at the corners of the Brillouin zone. A physical realization of such systems may bring the elusive spin field effect transistor closer to realization. The properties of spin transport in graphene have received intense interest ever since it was demonstrated that spin coherence lengths are in the micrometer range [39]. Injection and detection of electrons with different spin orientations is important in the context of a recently proposed experiment, in which Cooper pairs from a superconducting electrode are separated into two opposite graphene strips. Ferromagnetic contacts are then attached to the end of these strips, permitting the measurement of the electrons' spin state, thus offering to perform experiments with entangled electron states in a transport experiment.

Last but not least, the study of adsorption, intercalation processes on graphene itself, is a promising field for which only few reports are available at present. Functionalization of graphene is clearly an important issue, also in the context of doping and for sensing applications, but equally exciting is the prospect of using adsorption and self-organization processes of complex organic molecules on metal surfaces to create various "lower-than-two" dimensional graphene-like structures of the wire and dot type. The wealth of molecules with benzene ring segments provided by organic chemistry in ever larger numbers offers the possibility to prepare highquality graphene "nanoribbons", and first examples have already been described in the literature.

Progress in the field of graphene research, and also in studies of graphene on metals, is so rapid that the present overview can only give a glimpse of the current status, and may have to be updated within a year or so. The rapid pace of progress is a demonstration of the maturity of the way in which the experimental probes and their interpretation are mastered, and it is matched by the theoretical understanding of properties and processes at the surface and interface of graphene. Investigating graphene and carbon-related structures on metals holds great promise for novel and, from a basic science and applications-oriented perspective, exciting discoveries. 
Acknowledgements We thank all co-workers and collaborators for their contributions to this work, in particular, M. Weser, S. Böttcher, C. Enderlein, E. Voloshina, E. Goering, M. Sicot, P. Leicht, A. Zusan, O. Zander, A. S. Vinogradov, M. L. Ng, and S. Bouvron. This work has been supported by the European Science Foundation (ESF) under the EUROCORES Programme EuroGRAPHENE (Project "SpinGraph"). Y. D. acknowledges the financial support by the German Research Foundation (DFG) under project DE 1679/2-1. M. F. gratefully acknowledges the financial support by the Research Center "UltraQuantum" (Excellence Initiative), by the German Research Foundation (DFG) via the Collaborative Research Center (SFB) 767, and the BadenWürttemberg Stiftung. A. P. is grateful for the financial support from the Swedish Research Council.

\section{References}

1. A.K. Geim, K.S. Novoselov, Nat. Mater. 6, 183 (2007)

2. A.K. Castro Neto, F. Guinea, N.M.R. Peres, K.S. Novoselov, A.K. Geim, Rev. Mod. Phys. 81, 109 (2009)

3. A.K. Geim, Science 324, 1530 (2009)

4. Q. Yu, J. Lian, S. Siriponglert, H. Li, Y.P. Chen, S.-S. Pei, Appl. Phys. Lett. 93, 113103 (2008)

5. K.S. Kim, Y. Zhao, H. Jang, S.Y. Lee, J.M. Kim, K.S. Kim, J.-H. Ahn, P. Kim, J.-Y. Choi, B.H. Hong, Nature 457, 706 (2009)

6. X. Li, W. Cai, J. An, S. Kim, J. Nah, D. Yang, R. Piner, A. Velamakanni, I. Jung, E. Tutuc, S.K. Banerjee, L. Colombo, R.S. Ruoff, Science 324, 1312 (2009)

7. S. Bae, H. Kim, Y. Lee, X. Xu, J.-S. Park, Y. Zheng, J. Balakrishnan, T. Lei, H.R. Kim, Y.I. Song, Y.-J. Kim, K.S. Kim, B. Ozyilmaz, J.-H. Ahn, B.H. Hong, S. Iijima, Nat. Nanotechnol. 5, 574 (2010)

8. B.J. Kang, J.H. Mun, C. Y. Hwang, B.J. Cho, J. Appl. Phys. 106, 104309 (2009)

9. E. Sutter, P. Albrecht, P. Sutter, Appl. Phys. Lett. 95, 133109 (2009)

10. J. Bai, R. Cheng, F. Xiu, L. Liao, M. Wang, A. Shailos, K.L. Wang, Y. Huang, X. Duan, Nat. Nanotechnol. 5, 655 (2010)

11. J. Cai, P. Ruffieux, R. Jaafar, M. Bieri, T. Braun, S. Blankenburg, M. Muoth, A.P. Seitsonen, M. Saleh, X. Feng, K. Müllen, R. Fasel, Nature 466, 470 (2010)

12. T. Ohta, A. Bostwick, T. Seyller, K. Horn, E. Rotenberg, Science 313, 951 (2006)

13. Y. Zhang, T.-T. Tang, C. Girit, Z. Hao, M.C. Martin, A. Zettl, M.F. Crommie, Y.R. Shen, F. Wang, Nature 459, 820 (2009)

14. X. Wang, X. Li, L. Zhang, Y. Yoon, P.K. Weber, H. Wang, J. Guo, H. Dai, Science 324, 768 (2009)

15. S. Dutta, S.K. Pati, J. Phys. Chem. B 112, 1333 (2008)

16. Y. Mao, J. Yuan, J. Zhong, J. Phys.: Condens. Matter 20, 115209 (2008)

17. J. Wintterlin, M.L. Bocquet, Surf. Sci. 603, 1841 (2009)

18. P.A. Khomyakov, G. Giovannetti, P.C. Rusu, G. Brocks, J.V.D. Brink, P.J. Kelly, Phys. Rev. B 79, 195425 (2009)

19. E. Rotenberg, A. Bostwick, T. Ohta, J.L. McChesney, T. Seyller, K. Horn, Nat. Mater. 7, $258(2008)$

20. S.Y. Zhou, D.A. Siegel, A.V. Fedorov, F.E. Gabaly, A.K. Schmid, A.H.C. Neto, D.H. Lee, A. Lanzara, Nat. Mater. 7, 259 (2008)

21. G. Giovannetti, P.A. Khomyakov, G. Brocks, P.J. Kelly, J. van den Brink, Phys. Rev. B 76, 073103 (2007)

22. A. Shikin, G. Prudnikova, V. Adamchuk, F. Moresco, K. Rieder, Phys. Rev. B 62, 13202 (2000) 
23. Y.S. Dedkov, A.M. Shikin, V.K. Adamchuk, S.L. Molodtsov, C. Laubschat, A. Bauer, G. Kaindl, Phys. Rev. B 64, 035405 (2001)

24. Y.S. Dedkov, M. Fonin, U. Rüdiger, C. Laubschat, Appl. Phys. Lett. 93, 022509 (2008)

25. I. Gierz, C. Riedl, U. Starke, C. Ast, K. Kern, Nano Lett. 8, 4603 (2008)

26. C. Enderlein, Y.S. Kim, A. Bostwick, E. Rotenberg, K. Horn, New J. Phys. 12, 033014 (2010)

27. I. Gierz, T. Suzuki, R.T. Weitz, D.S. Lee, B. Krauss, C. Riedl, U. Starke, H. Hoechst, J.H. Smet, C. R. Ast, K. Kern, Phys. Rev. B 81, 235408 (2010)

28. K.T. Chan, J.B. Neaton, M.L. Cohen, Phys. Rev. B 77, 235430 (2008)

29. D. W. Boukhvalov, M.I. Katsnelson, J. Phys.: Condens. Matter. 21, 344205 (2009)

30. C. Coletti, C. Riedl, D.S. Lee, B. Krauss, L. Patthey, K. von Klitzing, J.H. Smet, U. Starke, Phys. Rev. B 81, 235401 (2010)

31. E.J.H. Lee, K. Balasubramanian, R.T. Weitz, M. Burghard, K. Kern, Nat. Nanotechnol. 3, $486(2008)$

32. F. Xia, T. Mueller, R. Golizadeh-Mojarad, M. Freitag, Y.-M. Lin, J. Tsang, V. Perebeinos, P. Avouris, Nano Lett. 9, 1039 (2009)

33. T. Mueller, F. Xia, M. Freitag, J. Tsang, P. Avouris, Phys. Rev. B 79, 245430 (2009)

34. B. Huard, N. Stander, J.A. Sulpizio, D. Goldhaber-Gordon, Phys. Rev. B 78, 121402 (2008)

35. V.M. Karpan, G. Giovannetti, P.A. Khomyakov, M. Talanana, A.A. Starikov, M. Zwierzycki, J. van den Brink, G. Brocks, P.J. Kelly, Phys. Rev. Lett. 99, 176602 (2007)

36. V.M. Karpan, P.A. Khomyakov, A.A. Starikov, G. Giovannetti, M. Zwierzycki, M. Talanana, G. Brocks, J.V.D. Brink, P.J. Kelly, Phys. Rev. B 78, 195419 (2008)

37. O.V. Yazyev, A. Pasquarello, Phys. Rev. B 80, 035408 (2009)

38. O.V. Yazyev, M.I. Katsnelson, Phys. Rev. Lett. 100, 047209 (2008)

39. N. Tombros, C. Jozsa, M. Popinciuc, H. T. Jonkman, B.J. van Wees, Nature 448, 571 (2007)

40. A. N'Diaye, S. Bleikamp, P. Feibelman, T. Michely, Phys. Rev. Lett. 97, 215501 (2006)

41. A.B. Preobrajenski, M.L. Ng, A.S. Vinogradov, N. Mårtensson, Phys. Rev. B 78, 073401 (2008)

42. S. Marchini, S. Guenther, J. Wintterlin, Phys. Rev. B 76, 075429 (2007)

43. D. Martoccia, P.R. Willmott, T. Brugger, M. Björck, S. Günther, C.M. Schlepütz, A. Cervellino, S.A. Pauli, B.D. Patterson, S. Marchini, J. Wintterlin, W. Moritz, T. Greber, Phys. Rev. Lett. 101, 126102 (2008)

44. A.L. Vazquez de Parga, F. Calleja, B. Borca, M.C.G. Passeggi, J.J. Hinarejos, F. Guinea, R. Miranda, Phys. Rev. Lett. 100, 1 (2008)

45. P.W. Sutter, J.-I. Flege, E.A. Sutter, Nature Mater. 7, 406 (2008)

46. P. Sutter, M.S. Hybertsen, J.T. Sadowski, E. Sutter, Nano Lett. 9, 2654 (2009)

47. B. Borca, S. Barja, M. Garnica, J.J. Hinarejos, A.L.V. de Parga, R. Miranda, F. Guinea, Semicond. Sci. Tech. 25, 034001 (2010)

48. H. Zhang, Q. Fu, Y. Cui, D. Tan, X. Bao, J. Phys. Chem. C 113, 8296 (2009)

49. Y. Pan, M. Gao, L. Huang, F. Liu, H.J. Gao, Appl. Phys. Lett. 95, 093106 (2009)

50. K. Donner, P. Jakob, J. Chem. Phys. 131, 164701 (2009)

51. M. Sicot, S. Bouvron, O. Zander, U. Ruediger, Y.S. Dedkov, M. Fonin, Appl. Phys. Lett. 96, 093115 (2010)

52. A. Nagashima, H. Itoh, T. Ichinokawa, C. Oshima, S. Otani, Phys. Rev. B 50, 4756 (1994)

53. C. Oshima, A. Nagashima, J Phys.: Condens. Matter 9, 1 (1997)

54. S. Hla, J. Vac. Sci. Technol. B 23, 1351 (2005)

55. D. Fujita and K. Sagisaka, Sci. Technol. Adv. Mater. 9, 013003 (2008)

56. J. Stöhr and M. Samant, J. Electr. Spectr. Rel. Phenom. 98, 189 (1999)

57. J. Stöhr, J. Magn. Magn. Mat. 200, 470 (1999)

58. B. Thole, P. Carra, F. Sette, G. van der Laan, Phys. Rev. Lett. 68, 1943 (1992)

59. P. Carra, B. Thole, M. Altarelli, X. Wang, Phys. Rev. Lett. 70, 694 (1993)

60. F. Reinert, S. Hüfner, New J. Phys. 7, 97 (2005) 
61. A. Damascelli, Z. Hussain, Z. Shen, Rev. Mod. Phys. 75, 473 (2003)

62. A. Damascelli, Phys. Scripta T109, 61 (2004)

63. P.D. Johnson, Rep. Prog. Phys. 60, 1217 (1997)

64. L. Isett, J. Blakely, Surf. Sci. 47, 645 (1975)

65. L. Isett, J. Blakely, Surf. Sci. 58, 397 (1976)

66. J. Shelton, H. Patil, J. Blakely, Surf. Sci. 43, 493 (1974)

67. M. Eizenberg, J. Blakely, Surf. Sci. 82, 228 (1979)

68. M. Eizenberg, J. Blakely, J. Chem. Phys. 71, 3467 (1979)

69. R. Rosei, M. Decrescenzi, F. Sette, C. Quaresima, A. Savoia, P. Perfetti, Phys. Rev. B 28, $1161(1983)$

70. Y. Gamo, A. Nagashima, M. Wakabayashi, M. Terai, C. Oshima, Surf. Sci. 374, 61 (1997)

71. E.N. Voloshina, Y.S. Dedkov, in Electronic and Magnetic Properties of the GrapheneFerromagnet Interfaces: Theory vs Experiment, ed. by S. Mikhailov. Physics and Applications of Graphene - Experiments (INTECH Education and Publishing, Vienna, 2010) ISBN 978953-307-217-3

72. R. Rosei, S. Modesti, F. Sette, C. Quaresima, A. Savoia, P. Perfetti, Solid State Commun. 46, $871(1983)$

73. A. Nagashima, N. Tejima, C. Oshima, Phys. Rev. B 50, 17487 (1994)

74. A. Grüneis, D. Vyalikh, Phys. Rev. B 77, 193401 (2008)

75. Y.S. Dedkov, M. Fonin, New J. Phys. 12, 125004 (2010)

76. G. Bertoni, L. Calmels, A. Altibelli, V. Serin, Phys. Rev. B 71, 075402 (2004)

77. Y.S. Dedkov, M. Fonin, C. Laubschat, Appl. Phys. Lett. 92, 052506 (2008)

78. Y.S. Dedkov, M. Fonin, U. Rüdiger, C. Laubschat, Phys. Rev. Lett. 100, 107602 (2008)

79. A. Grueneis, K. Kummer, D.V. Vyalikh, New J. Phys. 11, 073050 (2009)

80. M. Fuentes-Cabrera, M.I. Baskes, A.V. Melechko, M.L. Simpson, Phys. Rev. B 77, 035405 (2008)

81. G. Kalibaeva, R. Vuilleumier, S. Meloni, A. Alavi, G. Ciccotti, R. Rosei, J. Phys. Chem. B 110, 3638 (2006)

82. C. Klink, I. Stensgaard, F. Besenbacher, E. Laegsgaard, Surf. Sci. 342, 250 (1995)

83. D. Eom, D. Prezzi, K.T. Rim, H. Zhou, M. Lefenfeld, S. Xiao, C. Nuckolls, M.S. Hybertsen, T.F. Heinz, G.W. Flynn, Nano Lett. 9, 2844 (2009)

84. K. Yamamoto, M. Fukishima, T. Osaka, C. Oshima, Phys. Rev. B 45, 11358 (1992)

85. Y. Souzu, M. Tsukada, Surf. Sci. 326, 42 (1995)

86. M. Weser, Y. Rehder, K. Horn, M. Sicot, M. Fonin, A.B. Preobrajenski, E. N. Voloshina, E. Goering, Y.S. Dedkov, Appl. Phys. Lett. 96, 012504 (2010)

87. J. Rusz, A.B. Preobrajenski, M.L. Ng, N. A. Vinogradov, N. Mårtensson, O. Wessely, B. Sanyal, O. Eriksson, Phys. Rev. B 81, 073402 (2010)

88. O. Wessely, M. Katsnelson, O. Eriksson, Phys. Rev. Lett. 94, 167401 (2005)

89. G. Giovannetti, P.A. Khomyakov, G. Brocks, V.M. Karpan, J.V.D. Brink, P.J. Kelly, Phys. Rev. Lett. 101, 026803 (2008)

90. S. Andersson, M. Frank, A. Sandell, A. Giertz, B. Brena, P. Brühwiler, N. Mårtensson, J. Libuda, M. Baümer, H. Freund, J. Chem. Phys. 108, 2967 (1998)

91. F. B. de Mongeot, A. Toma, A. Molle, S. Lizzit, L. Petaccia, A. Baraldi, Phys. Rev. Lett. 97, 056103 (2006)

92. E. Shirley, L. Terminello, A. Santoni, F. Himpsel, Phys. Rev. B 51, 13614 (1995)

93. P. Srivastava, F. Wilhelm, A. Ney, M. Farle, H. Wende, N. Haack, G. Ceballos, K. Baberschke, Phys. Rev. B 58, 5701 (1998)

94. S. Dhesi, H. Dürr, G. van der Laan, E. Dudzik, N. Brookes, Phys. Rev. B 60, 12852 (1999)

95. A. Nesvizhskii, A. Ankudinov, J. Rehr, K. Baberschke, Phys. Rev. B 62, 15295 (2000)

96. K. Baberschke, Appl. Phys. A 62, 417 (1996)

97. D. Huang, H. Jeng, C. Chang, G. Guo, J. Chen, W. Wu, S. Chung, S. Shyu, C. Wu, H. Lin, C. Chen, Phys. Rev. B 66, 174440 (2002)

98. H. Mertins, S. Valencia, W. Gudat, P. Oppeneer, O. Zaharko, H. Grimmer, Europhys. Lett. 66, $743(2004)$ 
99. C. Sorg, N. Ponpandian, M. Bernien, K. Baberschke, H. Wende, R.Q. Wu, Phys. Rev. B 73, 064409 (2006)

100. Z.Y. Li, Z.Q. Yang, S. Qiao, J. Hu, R.Q. Wu, J. Phys.: Condens. Matter 23, 225502 (2011)

101. O. Rader, A. Varykhalov, J. Sanchez-Barriga, D. Marchenko, A. Rybkin, A.M. Shikin, Phys. Rev. Lett. 102, 057602 (2009)

102. H. Lyon, G. Somorjai, J. Chem. Phys. 46, 2539 (1967)

103. A. Morgan, G. Somorjai, Surf. Sci. 12, 405 (1968)

104. Z. Hu, D. Ogletree, M. van Hove, G. Somorjai, Surf. Sci. 180, 433 (1987)

105. T. Land, T. Michely, R. Behm, J. Hemminger, G. Comsa, Surf. Sci. 264, 261 (1992)

106. S. Entani, S. Ikeda, M. Kiguchi, K. Saiki, G. Yoshikawa, I. Nakai, H. Kondoh, T. Ohta, Appl. Phys. Lett. 88, 153126 (2006)

107. P. Sutter, J. Sadowski, E. Sutter, Phys. Rev. B 80, 245411 (2009)

108. M. Gao, Y. Pan, C. Zhang, H. Hu, R. Yang, H. Lu, J. Cai, S. Du, F. Liu, H.-J. Gao, Appl. Phys. Lett. 96, 053109 (2010)

109. B. Nieuwenhuys, D. Hagen, G. Rovida, G. Somorjai, Surf. Sci. 59, 155 (1976)

110. J. Coraux, A.T. N'Diaye, M. Engler, C. Busse, D. Wall, N. Buckanie, F.-J.M. zu Heringdorf, R. van Gastel, B. Poelsema, T. Michely, New J. Phys. 11, 023006 (2009)

111. E. Loginova, S. Nie, K. Thuermer, N.C. Bartelt, K.F. McCarty, Phys. Rev. B 80, 085430 (2009)

112. I. Pletikosić, M. Kralj, P. Pervan, R. Brako, J. Coraux, A. N'Diaye, C. Busse, T. Michely, Phys. Rev. Lett. 102, 056808 (2009)

113. P. Lacovig, M. Pozzo, D. Alfè, P. Vilmercati, A. Baraldi, S. Lizzit, Phys. Rev. Lett. 103, 166101 (2009)

114. R. Balog, B. Jorgensen, L. Nilsson, M. Andersen, E. Rienks, M. Bianchi, M. Fanetti, E. Laegsgaard, A. Baraldi, S. Lizzit, Z. Sljivancanin, F. Besenbacher, B. Hammer, T. G. Pedersen, P. Hofmann, L. Hornekaer, Nat. Mater. 9, 315 (2010)

115. J. Hamilton, J. Blakely, Surf. Sci. 91, 199 (1980)

116. J. Grant, T. Haas, Surf. Sci. 21, 76 (1970)

117. M. Fonin, M. Sicot, O. Zander, S. Bouvron, P. Leicht, U. Rudiger, M. Weser, Y.S. Dedkov, K. Horn, Nano Lett. (submitted) (2010)

118. F. Himpsel, K. Christmann, P. Heimann, D. Eastman, P. Feibelman, Surf. Sci. 115, L159 (1982)

119. M. Wu, Q. Xu, D. Goodman, J .Phys. Chem. 98, 5104 (1994)

120. B. Wang, M.L. Bocquet, S. Marchini, S. Guenther, J. Wintterlin, Phys. Chem. Chem. Phys. 10, 3530 (2008)

121. W. Moritz, B. Wang, M.L. Bocquet, T. Brugger, T. Greber, J. Wintterlin, S. Guenther, Phys. Rev. Lett. 104, 136102 (2010)

122. B. Lang, P. Legare, G. Maire, Surf. Sci. 47, 89 (1975)

123. C. McConville, D. Woodruff, S. Kevan, M. Weinert, J. Davenport, Phys. Rev. B 34, 2199 (1986)

124. T. Brugger, S. Guenther, B. Wang, J.H. Dil, M.-L. Bocquet, J. Osterwalder, J. Wintterlin, T. Greber, Phys. Rev. B 79, 045407 (2009)

125. M. Fonin, unpublished (2010)

126. J. Coraux, A.T. N'Diaye, C. Busse, T. Michely, Nano Lett. 8, 565 (2008)

127. A.T. N'Diaye, J. Coraux, T. N. Plasa, C. Busse, T. Michely, New J. Phys. 10, 043033 (2008)

128. A.T. N'Diaye, R. van Gastel, A.J. Martinez-Galera, J. Coraux, H. Hattab, D. Wall, F.-J.M. zu Heringdorf, M.H. von Hoegen, J.M. Gomez-Rodriguez, B. Poelsema, C. Busse, T. Michely, New J. Phys. 11, 113056 (2009)

129. R. van Gastel, A.T. N'Diaye, D. Wall, J. Coraux, C. Busse, N.M. Buckanie, F.-J.M. zu Heringdorf, M.H. von Hoegen, T. Michely, B. Poelsema, Appl. Phys. Lett. 95, 121901 (2009)

130. D. Martoccia, M. Bjoerck, C.M. Schlepuetz, T. Brugger, S.A. Pauli, B.D. Patterson, T. Greber, P.R. Willmott, New J. Phys. 12, 043028 (2010) 
131. M. Corso, W. Auwarter, M. Muntwiler, A. Tamai, T. Greber, J. Osterwalder, Science 303, 217 (2004)

132. H. Dil, J. Lobo-Checa, R. Laskowski, P. Blaha, S. Berner, J. Osterwalder, T. Greber, Science 319, 1824 (2008)

133. M. Sasaki, Y. Yamada, Y. Ogiwara, S. Yagyu, S. Yamamoto, Phys. Rev. B 61, 15653 (2000)

134. H. Ueta, M. Saida, C. Nakai, Y. Yamada, M. Sasaki, S. Yamamoto, Surf. Sci. 560, 183 (2004)

135. Y.S. Dedkov, H. Vita, M. Weser, K. Horn, M. Fonin, unpublished (2010)

136. P.J. Feibelman, Phys. Rev. B 77, 165419 (2008)

137. D. Farias, A. Shikin, K. Rieder, Y.S. Dedkov, J. Phys.: Condens. Matter. 11, 8453 (1999)

138. Y.S. Dedkov, M. Poygin, D. Vyalikh, A. Starodubov, A.M. Shikin, V.K. Adamchuk, arXiv:cond-mat/0304575v1 [cond-mat.mtrl-sci] (2003)

139. A. Varykhalov, J. Sanchez-Barriga, A.M. Shikin, C. Biswas, E. Vescovo, A. Rybkin, D. Marchenko, O. Rader, Phys. Rev. Lett. 101, 157601 (2008)

140. C. Binns, S. Baker, A. Keen, S. Mozley, C. Norris, H. Derbyshire, S. Bayliss, Phys. Rev. B 53, 7451 (1996)

141. R. Pfandzelter, G. Steierl, C. Rau, Phys. Rev. Lett. 74, 3467 (1995)

142. A. Shikin, M. Poigin, Y.S. Dedkov, S. Molodtsov, V. Adamchuk, Phys. Solid State 42, $1170(2000)$

143. A. Bostwick, T. Ohta, J. Mcchesney, K. Emtsev, T. Seyller, K. Horn, E. Rotenberg, New J. Phys. 9, 385 (2007)

144. A.T. N'Diaye, T. Gerber, C. Busse, J. Myslivecek, J. Coraux, T. Michely, New J. Phys. 11, 103045 (2009)

145. M.L. Ng, R. Balog, L. Hornekaer, A.B. Preobrajenski, N.A. Vinogradov, N. Mårtensson, K. Schulte, J. Phys. Chem. C 114, 18559 (2010)

146. J. O. Sofo, A.S. Chaudhari, G.D. Barber, Phys. Rev. B 75, 153401 (2007)

147. K.S. Novoselov, A.K. Geim, S.V. Morozov, D. Jiang, M.I. Katsnelson, I.V. Grigorieva, S.V. Dubonos, A.A. Firsov, Nature 438, 197 (2005)

148. Y. Zhang, Y.W. Tan, H.L. Stormer, P. Kim, Nature 438, 201 (2005) 